\title{
A 2D MODEL FOR HYDRODYNAMICS AND BIOLOGY COUPLING APPLIED TO ALGAE GROWTH SIMULATIONS
}

\author{
Olivier Bernard ${ }^{1}$, Anne-Céline Boulanger ${ }^{2,3}$, Marie-Odile Bristeau ${ }^{2,3}$ \\ AND JACQUES SAINTE-MARIE ${ }^{2,3,4}$
}

\begin{abstract}
Cultivating oleaginous microalgae in specific culturing devices such as raceways is seen as a future way to produce biofuel. The complexity of this process coupling non linear biological activity to hydrodynamics makes the optimization problem very delicate. The large amount of parameters to be taken into account paves the way for a useful mathematical modeling. Due to the heterogeneity of raceways along the depth dimension regarding temperature, light intensity or nutrients availability, we adopt a multilayer approach for hydrodynamics and biology. For free surface hydrodynamics, we use a multilayer Saint-Venant model that allows mass exchanges, forced by a simplified representation of the paddlewheel. Then, starting from an improved Droop model that includes light effect on algae growth, we derive a similar multilayer system for the biological part. A kinetic interpretation of the whole system results in an efficient numerical scheme. We show through numerical simulations in two dimensions that our approach is capable of discriminating between situations of mixed water or calm and heterogeneous pond. Moreover, we exhibit that a posteriori treatment of our velocity fields can provide lagrangian trajectories which are of great interest to assess the actual light pattern perceived by the algal cells and therefore understand its impact on the photosynthesis process.
\end{abstract}

Mathematics Subject Classification. 35Q35, 35Q92, 76D05, 76Z99.

Received September 3, 2012. Revised February 18, 2013.

Published online July 30, 2013.

\section{INTRODUCTION}

Recently, biofuel production from microalgae has proved to have a high potential for biofuel production $[18,49]$. Several studies have demonstrated that some microalgae species could store more than $50 \%$ of their dry weight in lipids under certain conditions of nitrogen deprivency $[18,42,50]$ leading to productivities in a range of order larger than terrestrial plants. In this article, we focus on microalgae cultivation in raceways (also called high rate ponds), whose hydrodynamics has been less studied than the photobioreactor culturing devices $[35,38,39,41,43]$. These annular shaped ponds of low depth $(10$ to $50 \mathrm{~cm}$ ) are mixed with a paddlewheel (see Fig. 3). Due to

Keywords and phrases. Hydrostatic Navier-Stokes equations, Saint-Venant equations, free surface stratified flows, multilayer system, kinetic scheme, droop model, raceway, hydrodynamics and biology coupling, algae growth.

1 Inria, team BioCore, BP93, 06902 Sophia-Antipolis Cedex, France. Olivier.Bernard@inria.fr

2 Inria, team ANGE, B.P. 105, 78153 Le Chesnay Cedex, France. Anne-Celine.Boulanger@inria.fr

3 UPMC Univ Paris 06, CNRS UMR 7598, Laboratoire Jacques-Louis Lions, 75005, Paris, France.

Marie-Odile.Bristeau@inria.fr

4 CETMEF, 2 boulevard Gambetta, 60200 Compiègne, France. Jacques. Sainte-Marie@inria.fr 
their inherent nonlinear and instationary properties, where hydrodynamics and biology are strongly coupled, managing and optimizing such processes is very tricky. Carrying out experiments on raceways is both expensive and time consuming. A model is thus a key tool to help in the optimal design of the process but also in its operation. The objective of this paper is to propose a new model describing the coupling between hydrodynamics and biology within a raceway.

The first dynamic model of a microalgal raceway pond was proposed by [46] assuming spatial homogeneity. The model was later consolidated by including time-discrete photoacclimation dynamics [47]. In parallel, other less elaborated models where proposed [25,26]. Latter on, the coupling of biology with hydrodynamics in raceways was studied [30,31], in order to optimize the raceway design. In [32], algae growth and transport is modelled and several tests are performed in order to study for instance the effect of the water height or temperature control on the algae concentration evolution. However, those studies might not guarantee some key properties such as mass balance. We claim that the model we develop satisfies crucial mathematical properties such as the conservation of biochemical variables or positivity of the water height.

Our approach is the following. Hydrodynamics is governed by the Navier-Stokes equations. But for reasons of robustness and computational costs, we use a multilayer Saint-Venant system that is a good approximation of the Navier-Stokes equations for dominated advection flows. The accuracy and the stability properties of the multilayer approach are demonstrated in $[4,5,44]$. We add for our problem a specific forcing mimicking the effect of a paddlewheel. For the growth of microalgae, we utilize an improved version of the Droop model [10,19,20]. The Droop model has been widely studied and validated $[11,12,34,45]$. It states that growth do not depend on the external nutrient concentration but on the internal cell quota of nutrients. The algae can indeed still grow a few days after exhaustion of the substrate thanks to their capacity to store nutrients. The enhanced Droop model [10] also takes into account the effect of light on phytoplankton. We then write the multi-layer version of the biological model, inspired from [4]. Afterwards, we give a kinetic interpretation of the whole system, allowing the derivation of a numerical scheme that has requested properties.

The outline is organized as follows. In Section 2 we describe and justify our system of partial differential equations. Afterwards, we derive the numerical scheme that we will use, based on a kinetic interpretation. We basically follow [4] and add the new state variables concerning biology. In Section 4 we explain how we model the raceway with the $2 \mathrm{D}$ code (the two dimensions being $(\mathrm{x}, \mathrm{z})$, respectively the length of the pond and the water depth) by adding periodic conditions. We also focus on the way agitation is introduced: we add a force mimicking a paddle-wheel and then we derive its contribution in the multilayer system, in the kinetic scheme and in the discrete scheme. We show that we have relevant results. Eventually, a last section deals with a Lagrangian approach of the algae tracking that could be useful regarding the elaboration of a better environment modeling for the algae.

\section{THE COUPLED MODEL}

We adopt and couple two continuous models, one for the representation of free surface hydrodynamics and the other for microalgae growth. We point out that it is a one way coupling: the biology is indeed advected and diffused by the water flow, but there is no retroaction of algae on the fluid. This is justified by the fact that the biological concentrations, even though greater in a raceway than they could be in an ocean or a lake, remain still much smaller than what is expected to change density or temperature of the raceway (let us recall for instance that the salinity of the seawater is around $37 \mathrm{~g} . \mathrm{L}^{-1}$ whereas we will never reach algae concentrations more than 1 or 2 g. $\left.L^{-1}\right)$.

\subsection{The hydrodynamics model}

Let us introduce first the hydrodynamics model in two dimensions. It will represent a free surface flow set into motion by a paddlewheel. As far as the modeling of geophysical free surface flows is concerned, two types of models and numerical techniques are usually investigated. On the one hand, when the flow is complex and no particular hypothesis can help simplify the model, finite differences or finite elements methods are used to solve 
the parabolic free surface Navier Stokes equations [17,29]. But this treatment raises computational issues and, as far as the authors know, can hardly guarantee good properties such as positivity of the water depth, tracer conservation, wet/dry interface treatment shocks, ... On the other hand, when the shallow water hypothesis can be considered, a vertically averaged version of Navier-Stokes equations is often used: the Saint-Venant system $[9,24]$. With an hyperbolic structure, numerical methods for shallow water flows consist in finite volume schemes and recent developments have allowed the recovery of properties such as the positivity of the water height. Let us cite the use of kinetic schemes introduced by Perthame [40] or the hydrostatic reconstruction by Audusse et al. in [6]. But those shallow water equations permit only the treatment of unstratified flows, which does not match our problem. We expect indeed to have high heterogeneities in our variables, due to the rapid light decline along the depth dimension. To sum up, because we want to tackle a complex realistic problem, we want to use a model that contains most of the phenomena expressed in Navier-Stokes equations, but which could be treated with Saint-Venant like tools, and this is what the multilayer Saint-Venant system provides. As stated in the introduction, efficiency of this new method has been proved in $[4,5,44]$. We allow us one hypothesis: the hydrostatic approximation. Whilst the vertical acceleration cannot be considered negligible around the paddlewheel, it is the case anywhere else in the raceway. Moreover the objective is not to reproduce the small scale perturbations of the paddlewheel over the flow but only to reproduce its first order effects. The paddlewheel applies a volumic force on the water:

$$
F_{\text {wheel }}(x, z, t)=F_{x}(x, z, t) \overrightarrow{e_{x}}+F_{z}(x, z, t) \overrightarrow{e_{z}}
$$

More details are given in Section 4 about the formulation of the force.

Therefore, we begin with the 2D hydrostatic Navier-Stokes equations with varying density, on which we plug the paddlewheel force:

$$
\begin{aligned}
& \frac{\partial \rho}{\partial t}+\frac{\partial \rho u}{\partial x}+\frac{\partial \rho w}{\partial z}=0, \\
& \frac{\partial \rho u}{\partial t}+\frac{\partial \rho u^{2}}{\partial x}+\frac{\partial \rho u w}{\partial z}+\frac{\partial p}{\partial x}=\frac{\partial \Sigma_{x x}}{\partial x}+\frac{\partial \Sigma_{x z}}{\partial z}+F_{x}(x, z, t), \\
& \frac{\partial p}{\partial z}=-\rho g+\frac{\partial \Sigma_{z x}}{\partial x}+\frac{\partial \Sigma_{z z}}{\partial z}+F_{z}(x, z, t),
\end{aligned}
$$

and we consider solutions of the equations for $t>t_{0}, \quad x \in \mathbb{R}, \quad z_{b}(x) \leq z \leq \eta(x, t)$, where $\eta(x, t)$ represents the free surface elevation, $\mathbf{u}=(u, w)^{T}$ the velocity vector, $p(x, z, t)$ is the pressure, $g$ the gravity acceleration and $\rho(T)$ is the water density, depending on an advected and diffused tracer $T$ basically representing the temperature and which satisfies the advection diffusion equation:

$$
\frac{\partial \rho T}{\partial t}+\frac{\partial \rho u T}{\partial x}+\frac{\partial \rho w T}{\partial z}=\mu_{T} \frac{\partial^{2} T}{\partial x^{2}}+\mu_{T} \frac{\partial^{2} T}{\partial z^{2}} .
$$

The flow height is $H=\eta-z_{b}$. The chosen form of the viscosity tensor is

$$
\Sigma_{x x}=2 \mu \frac{\partial u}{\partial x}, \quad \Sigma_{x z}=\mu \frac{\partial u}{\partial z}, \quad \Sigma_{z z}=2 \mu \frac{\partial w}{\partial z}, \quad \Sigma_{z x}=\mu \frac{\partial u}{\partial z}
$$

where $\mu$ is a dynamic viscosity.

Boundary conditions. The system (2.1)-(2.3) is completed with boundary conditions. The outward and upward unit normals to the free surface $\mathbf{n}_{s}$ and to the bottom $\mathbf{n}_{b}$ are given by

$$
\mathbf{n}_{s}=\frac{1}{\sqrt{1+\left(\frac{\partial \eta}{\partial x}\right)^{2}}}\left(\begin{array}{c}
-\frac{\partial \eta}{\partial x} \\
1
\end{array}\right), \quad \mathbf{n}_{b}=\frac{1}{\sqrt{1+\left(\frac{\partial z_{b}}{\partial x}\right)^{2}}}\left(\begin{array}{c}
-\frac{\partial z_{b}}{\partial x} \\
1
\end{array}\right) .
$$

Let $\Sigma_{T}$ be the total stress tensor with

$$
\Sigma_{T}=-p I_{d}+\left(\begin{array}{cc}
\Sigma_{x x} & \Sigma_{x z} \\
\Sigma_{z x} & \Sigma_{z z}
\end{array}\right) .
$$


Free surface conditions. At the free surface we have the kinematic boundary condition

$$
\frac{\partial \eta}{\partial t}+u_{s} \frac{\partial \eta}{\partial x}-w_{s}=0
$$

where the subscript $s$ denotes the value of the considered quantity at the free surface. Provided that the air viscosity is negligible, the continuity of stresses at the free boundary implies

$$
\Sigma_{T} \mathbf{n}_{s}=-p^{a} \mathbf{n}_{s}
$$

where $p^{a}=p^{a}(x, t)$ is a given function corresponding to the atmospheric pressure. In the following, we assume $p^{a}=0$.

Bottom conditions. The kinematic boundary condition is a classical no-penetration condition

$$
\mathbf{u}_{b} \cdot \mathbf{n}_{b}=0, \quad \text { or } \quad u_{b} \frac{\partial z_{b}}{\partial x}-w_{b}=0 .
$$

For the bottom stresses we consider a wall law

$$
\mathbf{t}_{b} \cdot \Sigma_{T} \mathbf{n}_{b}=\kappa \mathbf{u}_{b} \cdot \mathbf{t}_{b}
$$

where $\mathbf{t}_{b}$ a unit vector satisfying $\mathbf{t}_{b} \cdot \mathbf{n}_{b}=0$ and $\kappa$ is a friction coefficient.

\subsection{The biological model}

\subsubsection{Phytoplankton}

Microalgae have pigments to capture sunlight, which is turned into chemical energy during the photosynthesis process. They consume carbon dioxide, and release oxygen. Phytoplankton growth depends on the availability of carbon dioxide, sunlight, and nutrients. Required nutrients are of various types, but here we focus on inorganic nitrogen, such as nitrate, whose deprivency is known to stimulate lipid production [36]. We consider the combined influence of nitrate and light on phytoplankton growth. The nutrient limitation is taken into account by a Droop formulation [19] of the growth rate. The light effect (photosynthesis and photoinhibition) is represented using a classical formulation from [37] embedded in the model proposed by [10].

\subsubsection{Droop model with photoadaptation}

The Droop model represents the growth of an algal biomass $C^{1}$ using a nutrient of concentration $C^{3}$ (nitrate under the form $\mathrm{NO}_{3}$ ) in the medium. The concentration of particulate nitrogen (nitrogen contained in the algal biomass) is denoted $C^{2}$. Note that $C^{1}\left(g C . \mathrm{m}^{-3}\right), C^{2}\left(\mathrm{gN} \cdot \mathrm{m}^{-3}\right)$ and $C^{3}\left(\mathrm{gN} . \mathrm{m}^{-3}\right)$ are three transportable quantities. In order to reproduce the coupling between nutrient uptake rate $\lambda$ and growth rate $\mu$, Droop introduced the cell quota, $q(t, x)$, defined as the amount of internal nutrients per biomass unit: $q=\frac{C^{2}}{C^{1}}$. Microalgae growth rate thus depends on the intra-cellular quota:

$$
\mu(q)=\bar{\mu}\left(1-\frac{Q_{0}}{q}\right),
$$

where the constant $\bar{\mu}$ denotes the hypothetical growth rate for infinite quota, and $Q_{0}$ is the minimum internal nutrient quota required for growth.

The nitrate uptake rate is a function of the external nitrate [21]:

$$
\lambda\left(C^{3}\right)=\bar{\lambda} \frac{C^{3}}{C^{3}+K_{3}},
$$

where $K_{3}$ is the half saturation constant and $\bar{\lambda}$ the maximum uptake rate.

Finally, we will take into account both respiration and mortality, which are represented by a constant loss of the biomass with a factor $R$.

In line with [10], we modify this classical model in order to capture light and space variations. 
Light along the raceway depth. The previous model only included nutrient limited growth. It can be improved by introducing a new data in the system: the light intensity, which will depend on the quantity of water and biomass above the algae. Light is indeed attenuated by the chlorophyll concentration. This concentration can be linked to nitrogen through

$$
C h l=\gamma\left(I^{*}\right) C^{2}
$$

with

$$
\gamma\left(I^{*}\right)=\frac{k_{I^{*}}}{I^{*}+k_{I^{*}}}
$$

where $k_{I^{*}}$ is a constant, $I^{*}$ is the average light in the water column the day before(space and time average). $\gamma\left(I^{*}\right)$ is presumed constant over the day.

Let us assume that light intensity hitting the water surface is of the form

$$
I_{0}=I_{0}^{\max } \max (0, \sin (2 \pi t)),
$$

Then the intensity at depth $z$ can be described by

$$
I(z)=I_{0} \mathrm{e}^{-\psi\left(C^{2}, I^{*}, z\right)},
$$

where

$$
\psi\left(C^{2}, I^{*}, z\right)=\int_{0}^{z}\left(a \gamma\left(I^{*}\right) C^{2}(z)+b\right) \mathrm{d} z
$$

and $I_{0}^{\max }$ is a constant representing the maximum light intensity, $a$ and $b$ are also given constants.

The growth rate can then be computed to take into account light intensity, using Peeters and Eilers formalism $[27,37]$

$$
\mu(q, I)=\tilde{\mu} \frac{I}{I+K_{s I}+\frac{I^{2}}{K_{i I}}}\left(1-\frac{Q_{0}}{q}\right),
$$

with $\tilde{\mu}, K_{s I}, K_{i I}$ three given constants derived from dedicated experiments. Finally, a down regulation by the internal quota of the uptake rate must be included to avoid infinite substrate $\left(\mathrm{NO}_{3}\right)$ uptake in the dark

$$
\lambda\left(C^{3}, q\right)=\bar{\lambda} \frac{C^{3}}{C^{3}+K_{3}}\left(1-\frac{q}{Q_{l}}\right)
$$

where $Q_{l}$ is the maximum achievable quota.

Adding advection and diffusion, the biological system writes in the end

$$
\begin{aligned}
& \frac{\partial \rho C^{1}}{\partial t}+\frac{\partial \rho u C^{1}}{\partial x}+\frac{\partial \rho w C^{1}}{\partial z}=\mu_{C^{1}}\left(\frac{\partial^{2} C^{1}}{\partial x^{2}}+\frac{\partial^{2} C^{1}}{\partial z^{2}}\right)+\rho\left(\mu(q, I) C^{1}-R C^{1}\right), \\
& \frac{\partial \rho C^{2}}{\partial t}+\frac{\partial \rho u C^{2}}{\partial x}+\frac{\partial \rho w C^{2}}{\partial z}=\mu_{C^{2}}\left(\frac{\partial^{2} C^{2}}{\partial x^{2}}+\frac{\partial^{2} C^{2}}{\partial z^{2}}\right)+\rho\left(\lambda\left(C^{3}, q\right) C^{1}-R C^{2}\right), \\
& \frac{\partial \rho C^{3}}{\partial t}+\frac{\partial \rho u C^{3}}{\partial x}+\frac{\partial \rho w C^{3}}{\partial z}=\mu_{C^{3}}\left(\frac{\partial^{2} C^{3}}{\partial x^{2}}+\frac{\partial^{2} C^{3}}{\partial z^{2}}\right)-\rho \lambda\left(C^{3}, q\right) C^{1},
\end{aligned}
$$

where $q=\frac{C^{2}}{C^{1}}, \mu_{C^{1}}, \mu_{C^{2}}, \mu_{C^{3}}$ are diffusion coefficients and $(u, w)$ are the fluid velocities along $x$ and $z$ direction. 


\section{Multilayer model, Kinetic interpretation And Numerical SCHEME}

\subsection{Vertical space discretization: the multilayer model}

The next step is the vertical discretization of (2.1)-(2.3) and (2.14)-(2.16) in order to obtain a multilayer system. For (2.1)-(2.3), such a derivation has already been performed in $[4,5]$, but in our case we have a source term representing the paddlewheel effect in equations (2.2) and (2.3). Since the resulting vertically discretized equations are not straightforward from those two previous papers, we detail it here. However, for the sake of simplicity, we choose to omit the viscosity terms. The spatial discretization of the multilayer viscous terms can be found in [5], Section 3.

Moreover, we will use the classical equation of state relating the density and the tracer identified here with the temperature

$$
\rho(T)=\rho_{0}\left(1-\alpha\left(T-T_{0}\right)^{2}\right),
$$

with $T_{0}=4^{\circ} \mathrm{C}, \alpha=6.6310^{-6} \mathrm{C}^{2}$ and $\rho_{0}=10^{3} \mathrm{~kg} \cdot \mathrm{m}^{-3}$. In the range of temperatures that we plan to use, it is easy to check that density variations are small. Hence we use the Boussinesq assumption which states that those density variations are taken into account in the gravitational force only. In any other equation the density is supposed to be $\rho_{0}$. Eventually, we end up with the hydrodynamic system

$$
\begin{aligned}
& \frac{\partial u}{\partial x}+\frac{\partial w}{\partial z}=0 \\
& \frac{\partial u}{\partial t}+\frac{\partial u^{2}}{\partial x}+\frac{\partial u w}{\partial z}+\frac{1}{\rho_{0}} \frac{\partial p}{\partial x}=\frac{1}{\rho_{0}} F_{x}(x, z, t) \\
& \frac{\partial p}{\partial z}=-\rho g+F_{z}(x, z, t) \\
& \frac{\partial T}{\partial t}+\frac{\partial u T}{\partial x}+\frac{\partial w T}{\partial z}=0
\end{aligned}
$$

with $\rho=\rho(T)$ given by (3.1) and the biological system

$$
\begin{aligned}
& \frac{\partial C^{1}}{\partial t}+\frac{\partial u C^{1}}{\partial x}+\frac{\partial w C^{1}}{\partial z}=\mu(q, I) C^{1}-R C^{1}, \\
& \frac{\partial C^{2}}{\partial t}+\frac{\partial u C^{2}}{\partial x}+\frac{\partial w C^{2}}{\partial z}=\lambda\left(C^{3}, q\right) C^{1}-R C^{2}, \\
& \frac{\partial C^{3}}{\partial t}+\frac{\partial u C^{3}}{\partial x}+\frac{\partial w C^{3}}{\partial z}=-\lambda\left(C^{3}, q\right) C^{1},
\end{aligned}
$$

with $q=\frac{C^{2}}{C^{1}}$.

The process to obtain the multilayer system is described below. It is basically a Galerkin approximation of the variables followed by a vertical integration of the equations. The interval $\left[z_{b}, \eta\right]$ is divided into $N$ layers $\left\{L_{\alpha}\right\}_{\alpha \in\{1, \ldots, N\}}$ of thickness $l_{\alpha} H(x, t)$ where each layer $L_{\alpha}$ corresponds to the points satisfying $z \in L_{\alpha}(x, t)=$ $\left[z_{\alpha-1 / 2}, z_{\alpha+1 / 2}\right]$ with

$$
\left\{\begin{array}{l}
z_{\alpha+1 / 2}(x, t)=z_{b}(x, t)+\sum_{j=1}^{\alpha} l_{j} H(x, t), \\
h_{\alpha}(x, t)=z_{\alpha+1 / 2}(x, t)-z_{\alpha-1 / 2}(x, t)=l_{\alpha} H(x, t), \quad \alpha \in[0, \ldots, N]
\end{array}\right.
$$

with $l_{j}>0, \quad \sum_{j=1}^{N} l_{j}=1$.

Now let us consider the space $\mathbb{P}_{0, H}^{N, t}$ of piecewise constant functions defined by

$$
\mathbb{P}_{0, H}^{N, t}=\left\{\mathbb{I}_{z \in L_{\alpha}(x, t)}(z), \quad \alpha \in\{1, \ldots, N\}\right\},
$$


where $\mathbb{I}_{z \in L_{\alpha}(x, t)}(z)$ is the characteristic function of the interval $L_{\alpha}(x, t)$. Using this formalism, the projection of $u, w$ and $T$ onto $\mathbb{P}_{0, H}^{N, t}$ is a piecewise constant function defined by

$$
X^{N}\left(x, z,\left\{z_{\alpha}\right\}, t\right)=\sum_{\alpha=1}^{N} \mathbb{I}_{\left[z_{\alpha-1 / 2}, z_{\alpha+1 / 2}\right]}(z) X_{\alpha}(x, t),
$$

for $X \in(u, w, T)$. The density $\rho=\rho(T)$ inherits a discretization from the previous relation with

$$
\rho^{N}\left(x, z,\left\{z_{\alpha}\right\}, t\right)=\sum_{\alpha=1}^{N} \mathbb{I}_{\left[z_{\alpha-1 / 2}, z_{\alpha+1 / 2}\right]}(z) \rho\left(T_{\alpha}(x, t)\right) .
$$

We have the following result.

Proposition 3.1. The weak formulation of equations (3.2)-(3.5) on $\mathbb{P}_{0, H}^{N, t}$ leads to a system of the form

$$
\begin{aligned}
\sum_{\alpha=1}^{N} \frac{\partial l_{\alpha} H}{\partial t}+\sum_{\alpha=1}^{N} \frac{\partial l_{\alpha} H u_{\alpha}}{\partial x} & =0 \\
\frac{\partial h_{\alpha} u_{\alpha}}{\partial t}+\frac{\partial}{\partial x}\left(h_{\alpha} u_{\alpha}^{2}+h_{\alpha} p_{\alpha}\right)= & u_{\alpha+1 / 2} G_{\alpha+1 / 2}-u_{\alpha-1 / 2} G_{\alpha-1 / 2} \\
& +\frac{1}{\rho_{0}}\left(\frac{\partial z_{\alpha+1 / 2}}{\partial x} p_{\alpha+1 / 2}-\frac{\partial z_{\alpha-1 / 2}}{\partial x} p_{\alpha-1 / 2}\right) \\
& +\frac{1}{\rho_{0}} \int_{z_{\alpha-1 / 2}}^{z_{\alpha+1 / 2}} F_{x}(x, z, t) \mathrm{d} z \\
\frac{\partial h_{\alpha} T_{\alpha}}{\partial t}+\frac{\partial}{\partial x}\left(h_{\alpha} u_{\alpha} T_{\alpha}\right)= & T_{\alpha+1 / 2} G_{\alpha+1 / 2}-T_{\alpha-1 / 2} G_{\alpha-1 / 2}, \\
& \alpha \in[1, \ldots, N] .
\end{aligned}
$$

with

$$
\begin{aligned}
& G_{\alpha+1 / 2}=\frac{\partial z_{\alpha+1 / 2}}{\partial t}+u_{\alpha+1 / 2} \frac{\partial z_{\alpha+1 / 2}}{\partial x}-w_{\alpha+1 / 2} \\
& G_{1 / 2}=G_{N+1 / 2}=0 .
\end{aligned}
$$

The definitions of $p_{\alpha}, p_{\alpha+1 / 2}, u_{\alpha+1 / 2}, T_{\alpha+1 / 2}$ are given in the following proof.

Proof. Since the demonstration for part of this proposal can be found in $[4,5]$, we will not detail it here. However, we will focus on the integration of the agitation terms in this multilayer system.

The horizontal term. The horizontal term, in the $\mathrm{x}$-projection of the momentum equation will be handled simply: since we plan to use an expression of the force that can be integrated analytically (see 4.2.2), we just add the integrated force in the right-hand-side of (3.13)

$$
+\int_{z_{\alpha-1 / 2}}^{z_{\alpha+1 / 2}} F_{x}(x, z, t) \mathrm{d} z, \quad \alpha \in[1, \ldots, N] .
$$

The vertical term. The handling of this term will require more steps. First of all, let us recall that in the situation where no additional source terms are present, (3.4) is used to compute the piecewise continuous state variable $p$, which is then introduced in (3.3) (see [4]). We basically proceed the same way.

From (3.4), we get:

$$
p(x, z, t)=g \int_{z}^{\eta} \rho\left(x, z^{\prime}, t\right) \mathrm{d} z^{\prime}-\int_{z}^{\eta} F_{z}\left(x, z^{\prime}, t\right) \mathrm{d} z^{\prime}
$$


If we project it on $\mathbb{P}_{0, H}^{N, t}$, we get for a $z$ in layer $\alpha$

$$
p(x, z, t)=g\left(\sum_{j=\alpha+1}^{N} \rho_{j} h_{j}+\rho_{\alpha}\left(z_{\alpha+1 / 2}-z\right)\right)-\int_{z}^{\eta} F_{z}\left(x, z^{\prime}, t\right) \mathrm{d} z^{\prime} .
$$

For the vertical integration of (3.3) we need to compute:

$$
\int_{z_{\alpha-1 / 2}}^{z_{\alpha+1 / 2}} \frac{\partial p}{\partial x} \mathrm{~d} z=\frac{\partial h_{\alpha} p_{\alpha}}{\partial x}-\frac{\partial z_{\alpha+1 / 2}}{\partial x} p_{\alpha+1 / 2}+\frac{\partial z_{\alpha-1 / 2}}{\partial x} p_{\alpha-1 / 2}
$$

Since

$$
p_{\alpha}=\frac{1}{h_{\alpha}} \int_{z_{\alpha-1 / 2}}^{z_{\alpha+1 / 2}} p(x, z, t) \mathrm{d} z, \quad p_{\alpha+1 / 2}=p\left(x, z_{\alpha+1 / 2}, t\right),
$$

we see that the vertical agitation force has an effect in $p$ through (3.3). Let us precise the expressions of $p_{\alpha}(x, t)$, $p_{\alpha+1 / 2}(x, t)$ and $p_{\alpha-1 / 2}(x, t)$.

$$
\begin{aligned}
p_{\alpha}(x, t) & =\frac{1}{h_{\alpha}} \int_{z_{\alpha-1 / 2}}^{z_{\alpha+1 / 2}} p(x, z, t) \mathrm{d} z \\
& =g\left(\frac{\rho_{\alpha} h_{\alpha}}{2}+\sum_{j=\alpha+1}^{N} \rho_{j} h_{j}\right)-\frac{1}{h_{\alpha}} \int_{z_{\alpha-1 / 2}}^{z_{\alpha+1 / 2}} \int_{z}^{\eta} F_{z}\left(x, z^{\prime}, t\right) \mathrm{d} z^{\prime} \mathrm{d} z, \\
p_{\alpha+1 / 2}(x, t) & =g \sum_{j=\alpha+1}^{N} \rho_{j} h_{j}-\int_{z_{\alpha+1 / 2}}^{\eta} F_{z}\left(x, z^{\prime}, t\right) \mathrm{d} z^{\prime}, \\
p_{\alpha-1 / 2}(x, t) & =g \sum_{j=\alpha}^{N} \rho_{j} h_{j}-\int_{z_{\alpha-1 / 2}}^{\eta} F_{z}\left(x, z^{\prime}, t\right) \mathrm{d} z^{\prime} .
\end{aligned}
$$

The velocities $u_{\alpha+1 / 2}, \alpha=1, \ldots, N-1$ are obtained using an upwinding with respect to the direction of the mass exchange:

$$
u_{\alpha+1 / 2}= \begin{cases}u_{\alpha} & \text { if } G_{\alpha+1 / 2} \geq 0 \\ u_{\alpha+1} & \text { if } G_{\alpha+1 / 2}<0 .\end{cases}
$$

We proceed in the same way for the tracer:

$$
T_{\alpha+1 / 2}= \begin{cases}T_{\alpha} & \text { if } G_{\alpha+1 / 2} \geq 0 \\ T_{\alpha+1} & \text { if } G_{\alpha+1 / 2}<0\end{cases}
$$

In Proposition 3.1 the vertical velocity $w$ no more appears, but we can derive relations for the discrete layer values of this variable by performing the Galerkin approximation of the continuity equation (3.2) multiplied by $z$. This leads to

$$
\frac{\partial}{\partial t}\left(\frac{z_{\alpha+1 / 2}^{2}-z_{\alpha-1 / 2}^{2}}{2}\right)+\frac{\partial}{\partial x}\left(\frac{z_{\alpha+1 / 2}^{2}-z_{\alpha-1 / 2}^{2}}{2} u_{\alpha}\right)=h_{\alpha} w_{\alpha}+z_{\alpha+1 / 2} G_{\alpha+1 / 2}-z_{\alpha-1 / 2} G_{\alpha-1 / 2},
$$

where the $w_{\alpha}, \alpha=1, \ldots, N$, are the components of the Galerkin approximation of $w$ on $\mathbb{P}_{0, H}^{N, t}$, see (3.10). Since all the quantities except $w_{\alpha}$ appearing in equation (3.27) are already defined by (3.12), (3.13), (3.15), (3.16), relation (3.27) allows obtaining the values $w_{\alpha}$ by post-processing. Note that we use the relation (3.27) rather than the divergence free condition for stability purposes. We refer the reader to $[4,44]$ for more details. 
Proposition 3.2. Multilayer version of the Droop model with photoacclimation. The weak formulation of equations (3.6)-(3.8) on $\mathbb{P}_{0, H}^{N, t}$ leads to a system of the form

$$
\begin{aligned}
\frac{\partial h_{\alpha} C_{\alpha}^{1}}{\partial t}+\frac{\partial}{\partial x}\left(h_{\alpha} C_{\alpha}^{1} u_{\alpha}\right) & =C_{\alpha+1 / 2}^{1} G_{\alpha+1 / 2}-C_{\alpha-1 / 2}^{1} G_{\alpha-1 / 2} \\
& +h_{\alpha}\left(\mu\left(q_{\alpha}, I_{\alpha}\right) C_{\alpha}^{1}-R C_{\alpha}^{1}\right), \\
\frac{\partial h_{\alpha} C_{\alpha}^{2}}{\partial t}+\frac{\partial}{\partial x}\left(h_{\alpha} C_{\alpha}^{2} u_{\alpha}\right) & =C_{\alpha+1 / 2}^{2} G_{\alpha+1 / 2}-C_{\alpha-1 / 2}^{2} G_{\alpha-1 / 2} \\
& +h_{\alpha}\left(\lambda\left(C_{\alpha}^{3}, q_{\alpha}\right) C_{\alpha}^{1}-R C_{\alpha}^{2}\right), \\
\frac{\partial h_{\alpha} C_{\alpha}^{3}}{\partial t}+\frac{\partial}{\partial x}\left(h_{\alpha} C_{\alpha}^{3} u_{\alpha}\right) & =C_{\alpha+1 / 2}^{3} G_{\alpha+1 / 2}-C_{\alpha-1 / 2}^{3} G_{\alpha-1 / 2} \\
& -h_{\alpha} \lambda\left(C_{\alpha}^{3}, q_{\alpha}\right) C_{\alpha}^{1}, \quad \alpha \in[1, \ldots, N],
\end{aligned}
$$

with $q_{\alpha}=\frac{C_{\alpha}^{2}}{C_{\alpha}^{1}}$ and $C_{\alpha+1 / 2}^{j}, j=1 \ldots 3$ defined through the same upwinding as $u_{\alpha+1 / 2}$ and $T_{\alpha+1 / 2}($ see 3.25, 3.26).

Proof. As for equations (3.2)-(3.5), we use a Galerkin approximation of the biological variables on $\mathbb{P}_{0, H}^{N, t}$. The Galerkin approximation on $\mathbb{P}_{0, H}^{N, t}$ allows to write

$$
Y^{N}\left(x, z,\left\{z_{\alpha}\right\}, t\right)=\sum_{\alpha=1}^{N} 1_{\left[z_{\alpha-1 / 2}, z_{\alpha+1 / 2}\right]}(z) Y_{\alpha}(x, t),
$$

for $Y \in\left(C^{1}, C^{2}, C^{3}\right)$. Then we perform an integration of equations (3.6)-(3.8) over the layer $\alpha$. Let us do it term by term.

$$
\int_{z_{\alpha-1 / 2}}^{z_{\alpha+1 / 2}} \frac{\partial Y}{\partial t} \mathrm{~d} z=\frac{\partial h_{\alpha} Y_{\alpha}}{\partial t}-\left(Y_{\alpha+1 / 2} \frac{\partial z_{\alpha+1 / 2}}{\partial t}-Y_{\alpha-1 / 2} \frac{\partial z_{\alpha-1 / 2}}{\partial t}\right)
$$

For the transport terms it yields:

$$
\begin{aligned}
& \int_{z_{\alpha-1 / 2}}^{z_{\alpha+1 / 2}} \frac{\partial u Y}{\partial x} \mathrm{~d} z=\frac{\partial u_{\alpha} h_{\alpha} Y_{\alpha}}{\partial x}-\left(u_{\alpha+1 / 2} Y_{\alpha+1 / 2} \frac{\partial z_{\alpha+1 / 2}}{\partial x}-u_{\alpha-1 / 2} Y_{\alpha-1 / 2} \frac{\partial z_{\alpha-1 / 2}}{\partial x}\right) \\
& \int_{z_{\alpha-1 / 2}}^{z_{\alpha+1 / 2}} \frac{\partial w Y}{\partial z} \mathrm{~d} z=w_{\alpha+1 / 2} Y_{\alpha+1 / 2}-w_{\alpha-1 / 2} Y_{\alpha-1 / 2}
\end{aligned}
$$

For the reaction terms we get:

$$
\begin{gathered}
\int_{z_{\alpha-1 / 2}}^{z_{\alpha+1 / 2}}\left(\mu(q, I) C^{1}-R C^{1}\right) \mathrm{d} z=h_{\alpha}\left(\mu\left(q_{\alpha}, I_{\alpha}\right) C_{\alpha}^{1}-R C_{\alpha}^{1}\right), \\
\int_{z_{\alpha-1 / 2}}^{z_{\alpha+1 / 2}}\left(\lambda\left(C^{3}, q\right) C^{1}-R C^{2}\right) \mathrm{d} z=h_{\alpha}\left(\lambda\left(C_{\alpha}^{3}, q_{\alpha}\right) C_{\alpha}^{1}-R C_{\alpha}^{2}\right), \\
\int_{z_{\alpha-1 / 2}}^{z_{\alpha+1 / 2}}-\lambda\left(C^{3}, q\right) X \mathrm{~d} z=-h_{\alpha}\left(\lambda\left(C_{\alpha}^{3}, q_{\alpha}\right) C_{\alpha}^{1}\right),
\end{gathered}
$$

where

$$
q=\frac{C^{2}}{C^{1}} \text { and } q_{\alpha}=\frac{C_{\alpha}^{2}}{C_{\alpha}^{1}}
$$

Using the notation (3.15) and (3.16) we recover equations (3.28)-(3.30). 


\subsection{Kinetic interpretation}

The kinetic approach consists in linking the behaviour of some macroscopic fluid systems - Euler or NavierStokes equations, Saint-Venant system - with Boltzmann type kinetic equations. Boltzmann equation was first introduced in gas dynamics. It represents the evolution of a density of particles in a gas. Kinetic schemes have been widely used for the resolution of Euler equations [14,33]. Given the analogy between Euler and SaintVenant equations, recent work has been carried out to adapt those schemes to the shallow water systems ([1]). The first step is the introduction of fictitious particles, the definition of a density of particles and the equation governing its evolution.

The process to obtain the kinetic interpretation of the multilayer hydrodynamic model (3.12)-(3.14) is similar to the one used in [4]. For that reason we will only detail the kinetic interpretation of the multilayer biological system (3.28)-(3.30).

For a given layer $\alpha$, a distribution function $M_{\alpha}(x, t, \xi)$ of fictitious particles with microscopic velocity $\xi$ is introduced to obtain a linear kinetic equation equivalent to the macroscopic model.

Let us introduce a real function $\chi$ defined on $\mathbb{R}$, compactly supported and which have the following properties

$$
\left\{\begin{array}{l}
\chi(-w)=\chi(w) \geq 0 \\
\int_{\mathbb{R}} \chi(w) \mathrm{d} w=\int_{\mathbb{R}} w^{2} \chi(w) \mathrm{d} w=1
\end{array}\right.
$$

Now let us construct a density of particles $M_{\alpha}(x, t, \xi)$ defined by a Gibbs equilibrium: the microscopic density of particles present at time $t$, in the layer $\alpha$, at the abscissa $x$ and with velocity $\xi$ given by

$$
M_{\alpha}=\frac{h_{\alpha}(x, t)}{c_{\alpha}} \chi\left(\frac{\xi-u_{\alpha}(x, t)}{c_{\alpha}}\right)
$$

with

$$
c_{\alpha}^{2}=p_{\alpha},
$$

and $p_{\alpha}$ defined by (3.22).

Likewise, we define $N_{\alpha+1 / 2}(x, t, \xi)$ by

$$
N_{\alpha+1 / 2}(x, t, \xi)=G_{\alpha+1 / 2}(x, t) \delta\left(\xi-u_{\alpha+1 / 2}(x, t)\right),
$$

for $\alpha=0, \ldots, N$ and where $\delta$ denotes the Dirac distribution.

The quantities $G_{\alpha+1 / 2}, 0 \leq \alpha \leq N$ represent the mass exchanges between layers $\alpha$ and $\alpha+1$, they are defined in (3.15) and satisfy the conditions (3.16), so $N_{1 / 2}$ and $N_{N+1 / 2}$ also satisfy

$$
N_{1 / 2}(x, t, \xi)=N_{N+1 / 2}(x, t, \xi)=0 .
$$

For the Droop variables, we have the equilibria

$$
\begin{aligned}
& U_{\alpha}^{j}(x, t, \xi)=C_{\alpha}^{j}(x, t) M_{\alpha}(x, t, \xi), \quad \alpha=1, \ldots, N, \quad j=1, \ldots, 3 \\
& V_{\alpha+1 / 2}^{j}(x, t, \xi)=C_{\alpha+1 / 2}^{j}(x, t) N_{\alpha+1 / 2}(x, t, \xi), \quad \alpha=0, \ldots, N, \quad j=1, \ldots, 3 .
\end{aligned}
$$


With the previous definitions we write a kinetic representation of the multilayer biological system (3.28)(3.30) without biological reaction terms and we have the following proposition:

Proposition 3.3. The functions $\left(C^{j}\right)$ are strong solutions of the system (3.28)-(3.30) without reaction terms if and only if the set of equilibria $\left\{U_{\alpha}^{j}(x, t, \xi)\right\}_{\alpha=1}^{N}$ are solutions of the kinetic equations

$$
\frac{\partial U_{\alpha}^{j}}{\partial t}+\xi \frac{\partial U_{\alpha}^{j}}{\partial x}-V_{\alpha+1 / 2}^{j}+V_{\alpha-1 / 2}^{j}=Q_{U_{\alpha}^{j}}
$$

for $\alpha=1, \ldots, N, j=1 \ldots 3$ with $\left\{N_{\alpha+1 / 2}(x, t, \xi), V_{\alpha+1 / 2}^{j}(x, t, \xi)\right\}_{\alpha=0}^{N}$ satisfying (3.40)-(3.43).

The quantities $Q_{U_{\alpha}^{j}}=Q_{U_{\alpha}^{j}}(x, t, \xi)$ are "collision terms" equal to zero at the macroscopic level i.e. which satisfy for a.e. values of $(x, t)$

$$
\int_{\mathbb{R}} Q_{U_{\alpha}^{j}} \mathrm{~d} \xi=0, \quad \int_{\mathbb{R}} \xi Q_{U_{\alpha}^{j}} \mathrm{~d} \xi=0, \quad \text { and } \quad \int_{\mathbb{R}} \xi^{2} Q_{U_{\alpha}^{j}} \mathrm{~d} \xi=0 .
$$

Proof. Using the definitions (3.39), (3.42) and the properties of the function $\chi$, we have

$$
l_{\alpha} H C_{\alpha}^{j}=\int_{\mathbb{R}} U_{\alpha}^{j}(x, t, \xi) \mathrm{d} \xi, \quad l_{\alpha} H C_{\alpha}^{j} u_{\alpha}=\int_{\mathbb{R}} \xi U_{\alpha}^{j}(x, t, \xi) \mathrm{d} \xi .
$$

From the definition (3.40) of $N_{\alpha+1 / 2}$ we also have

$$
\int_{\mathbb{R}} N_{\alpha+1 / 2}(x, t, \xi) \mathrm{d} \xi=G_{\alpha+1 / 2},
$$

A simple integration in $\xi$ of the equations (3.44), always using (3.45), gives the biological equations (3.28)(3.30).

\subsection{The numerical scheme}

As in paragraph 3.2, we only detail the discretization of the biological part of the model. For the hydrodynamic part, the reader can refer to [ ]. Using the multilayer system obtained in Proposition 3.2 we end up with a system of the form

$$
\frac{\partial X}{\partial t}+\frac{\partial F(X)}{\partial x}=S_{e}(X)+S_{v, f}(X)+S_{b i o}(X)
$$

with $X=\left(k_{1}^{1}, \ldots, k_{N}^{1}, k_{1}^{2} \ldots k_{N}^{2}, k_{1}^{3} \ldots k_{N}^{3}\right)^{T}$ and $k_{\alpha}^{1}=l_{\alpha} H C_{\alpha}^{1}, k_{\alpha}^{2}=l_{\alpha} H C_{\alpha}^{2}, k_{\alpha}^{3}=l_{\alpha} H C_{\alpha}^{3}$. We denote $F(X)$ the flux of the conservative part, $S_{e}(X), S_{v, f}(X)$ and $S_{b i o}(X)$ the source terms, respectively the mass transfer, the viscous and friction effects and the biological reaction terms.

We introduce a $3 N \times 3 N$ matrix $\mathcal{K}(\xi)$ defined by $\mathcal{K}_{i, j}=\delta_{i, j}$ for $i, j=1, \ldots, N$ with $\delta_{i, j}$ the Kronecker symbol. Then, using Proposition 3.3, we can write

$$
\begin{array}{r}
X=\int_{\xi} \mathcal{K}(\xi)\left(\begin{array}{c}
U^{1}(\xi) \\
U^{2}(\xi) \\
U^{3}(\xi)
\end{array}\right) \mathrm{d} \xi, \quad F(X)=\int_{\xi} \xi \mathcal{K}(\xi)\left(\begin{array}{c}
U^{1}(\xi) \\
U^{2}(\xi) \\
U^{3}(\xi)
\end{array}\right) \mathrm{d} \xi \\
S_{e}(X)=\int_{\xi} \mathcal{K}(\xi)\left(\begin{array}{c}
V^{1}(\xi) \\
V^{2}(\xi) \\
V^{3}(\xi)
\end{array}\right) \mathrm{d} \xi
\end{array}
$$

with $U^{j}(\xi)=\left(U_{1}^{j}(\xi), \ldots, U_{N}^{j}(\xi)\right)^{T}$, and

$$
V(\xi)^{j}=\left(\begin{array}{c}
V_{3 / 2}^{j}(\xi)-V_{1 / 2}^{j}(\xi) \\
\vdots \\
V_{N+1 / 2}^{j}(\xi)-V_{N-1 / 2}^{j}(\xi)
\end{array}\right), \quad \forall j \in 1 . .3 .
$$

We refer to [5] for the computation of $S_{v, f}(X)$. 
To approximate the solution of (3.48) we use a finite volume framework. We assume that the computational domain is discretized by $I$ nodes $x_{i}$. We denote $C_{i}$ the cell of length $\Delta x_{i}=x_{i+1 / 2}-x_{i-1 / 2}$ with $x_{i+1 / 2}=$ $\left(x_{i}+x_{i+1}\right) / 2$. For the time discretization, we denote $t^{n}=\sum_{k<n} \Delta t^{k}$ where the time steps $\Delta t^{k}$ will be precised later through a CFL condition. We denote

$$
X_{i}^{n}=\left(k_{1, i}^{1, n}, \ldots, k_{N, i}^{1, n}, k_{1, i}^{2, n}, \ldots, k_{N, i}^{2, n}, k_{1, i}^{3, n}, \ldots, k_{N, i}^{3, n}\right)^{T}
$$

the approximate solution at time $t^{n}$ on the cell $C_{i}$ with $k_{\alpha, i}^{j, n}=l_{\alpha} H_{i}^{n} C_{\alpha, i}^{j, n}$.

\subsubsection{Time splitting}

For the time discretization, we apply a time splitting to the equation (3.48) and we write

$$
\begin{aligned}
& \frac{\tilde{X}^{n+1}-X^{n}}{\Delta t^{n}}+\frac{\partial F\left(X^{n}\right)}{\partial x}=S_{e}\left(X^{n}, \tilde{X}^{n+1}\right)+S_{b i o}\left(X^{n}\right), \\
& \frac{X^{n+1}-\tilde{X}^{n+1}}{\Delta t^{n}}-S_{v, f}\left(X^{n}, X^{n+1}\right)=0 .
\end{aligned}
$$

The conservative part of (3.51) is computed by an explicit kinetic scheme. The mass exchange terms are deduced from the kinetic interpretation. The biological reaction terms are not included in the kinetic interpretation but simply deduced from quadrature formulas. Eventually, the viscous and friction terms $S_{v, f}$ in (3.52) do not depend on the fluid density $\rho$. Thus, their vertical discretization and their numerical treatment do not differ from earlier works of the authors [5]. Due to potential dissipative effects, a semi-implicit scheme is adopted in the second step for reasons of stability.

\subsubsection{Discrete kinetic equation}

Starting from a piecewise constant approximation of the initial data, the general form of a finite volume discretization of system $(3.51)$ is

$$
\tilde{X}_{i}^{n+1}-X_{i}^{n}+\sigma_{i}^{n}\left[F_{i+1 / 2}^{n}-F_{i-1 / 2}^{n}\right]=\Delta t^{n} \mathcal{S}_{e, i}^{n+1 / 2}+\Delta t^{n} \mathcal{S}_{b i o}^{n},
$$

where $\sigma_{i}^{n}=\Delta t^{n} / \Delta x_{i}$ is the ratio between space and time steps and the numerical flux $F_{i+1 / 2}^{n}$ is an approximation of the exact flux estimated at point $x_{i+1 / 2}$.

In order to find a good expression of the numerical fluxes, we need to make an incursion in the microscopic scale. Therefore we denote the discrete particle density at time $n$, cell $i$ in the following manner:

$$
M_{\alpha, i}^{n}(\xi)=l_{\alpha} \frac{H_{i}^{n}}{c_{\alpha, i}^{n}} \chi\left(\frac{\xi-u_{\alpha, i}^{n}}{c_{\alpha, i}^{n}}\right), \quad \text { with } c_{\alpha, i}^{n}=\sqrt{p_{\alpha, i}^{n}}
$$

and following (3.22)

$$
p_{\alpha, i}^{n}=g\left(\frac{\rho_{\alpha, i}^{n} l_{\alpha} H_{i}^{n}}{2}+\sum_{j=\alpha+1}^{N} \rho_{j, i}^{n} l_{j} H_{i}^{n}\right)-\frac{1}{h_{\alpha}} \int_{z_{\alpha-1 / 2}}^{z_{\alpha+1 / 2}} \int_{z}^{\eta} F_{z}\left(x_{i}, z^{\prime}, t^{n}\right) \mathrm{d} z^{\prime} \mathrm{d} z .
$$

Then the equation (3.44) is discretized for each $\alpha$ by applying a simple upwind scheme

$$
g_{\alpha, i}^{j, n+1}(\xi)=U_{\alpha, i}^{j, n}(\xi)-\xi \sigma_{i}^{n}\left(U_{\alpha, i+1 / 2}^{j, n}(\xi)-U_{\alpha, i-1 / 2}^{j, n}(\xi)\right)+\Delta t^{n}\left(V_{\alpha+1 / 2, i}^{j, n+1 / 2}(\xi)-V_{\alpha-1 / 2, i}^{j, n+1 / 2}(\xi)\right),
$$

where

$$
U_{\alpha, i+1 / 2}^{j, n}= \begin{cases}U_{\alpha, i}^{j, n} & \text { if } \xi \geq 0 \\ U_{\alpha, i+1}^{j, n} & \text { if } \xi<0\end{cases}
$$


The quantity $g_{\alpha, i}^{j, n+1}$ is not an equilibrium but if we set

$$
l_{\alpha} H_{i}^{n+1} C_{\alpha, i}^{j, n+1}=\int_{\mathbb{R}} g_{\alpha, i}^{j, n+1}(\xi) \mathrm{d} \xi
$$

we recover the macroscopic quantities at time $t^{n+1}$.

\subsubsection{Numerical flux of the finite volume scheme}

In this section, we give some details for the computation of the fluxes introduced in the discrete equation (3.53). If we denote

$$
F_{i+1 / 2}^{n}=F\left(X_{i}^{n}, X_{i+1}^{n}\right)=F^{+}\left(X_{i}^{n}\right)+F^{-}\left(X_{i+1}^{n}\right),
$$

following (3.49), we define

$$
F^{-}\left(X_{i}^{n}\right)=\int_{\xi \in \mathbb{R}^{-}} \xi \mathcal{K}(\xi) U_{i}^{n}(\xi) \mathrm{d} \xi, \quad F^{+}\left(X_{i}^{n}\right)=\int_{\xi \in \mathbb{R}^{+}} \xi \mathcal{K}(\xi) U_{i}^{n}(\xi) \mathrm{d} \xi
$$

with $U_{i}^{n}(\xi)=\left(U_{1, i}^{n}(\xi), \ldots, U_{N, i}^{n}(\xi)\right)^{T}$.

More precisely the expression of $F^{+}\left(X_{i}\right)$ can be written

$$
F^{+}\left(X_{i}\right)=\left(F_{k_{1}^{1}}^{+}\left(X_{i}\right), \ldots, F_{k_{N}^{1}}^{+}\left(X_{i}\right), \quad F_{k_{1}^{2}}^{+}\left(X_{i}\right), \ldots, F_{k_{N}^{2}}^{+}\left(X_{i}\right), F_{k_{1}^{3}}^{+}\left(X_{i}\right), \ldots, F_{k_{N}^{3}}^{+}\left(X_{i}\right)\right)^{T},
$$

with

$$
F_{k_{\alpha}^{j}}^{+}\left(X_{i}\right)=C_{\alpha, i}^{j} l_{\alpha} H_{i} \int_{w \geq-\frac{u_{\alpha, i}}{c_{i}}}\left(u_{\alpha, i}+w c_{\alpha, i}\right) \chi(w) \mathrm{d} w .
$$

This kinetic method is interesting because it gives a very simple and natural way to propose a numerical flux through the kinetic interpretation. Indeed, choosing

$$
\chi(w)=\frac{1}{2 \sqrt{3}} 1_{|w| \leq \sqrt{3}}(w),
$$

the integration in (3.59) can be done analytically.

However, this method proved to be numerically diffusive. That is why in practice, following $[2,4]$, we rather introduce the upwinding in the biological equations according to the sign of the total mass flux. We introduce then new biological fluxes that we will use instead of (3.59)

$$
F_{k_{\alpha}^{j}}^{+}\left(X_{i}\right)=C_{\alpha, i+1 / 2}^{j} F_{h_{\alpha}}^{+}
$$

with

$$
F_{h_{\alpha}}^{+}=l_{\alpha} H_{i} \int_{w \geq-\frac{u_{\alpha, i}}{c_{i}}}\left(u_{\alpha, i}+w c_{\alpha, i}\right) \chi(w) \mathrm{d} w
$$

representing the mass flux in layer $\alpha$ at interface $i+1 / 2$ (see [4]) and the interfacial quantity being defined in the following manner

$$
C_{\alpha, i+1 / 2}^{j, n}= \begin{cases}C_{\alpha, i}^{j, n} & \text { if } F_{h_{\alpha}}^{+} \geq 0 \\ C_{\alpha, i+1}^{j, n} & \text { if } F_{h_{\alpha}}^{+}<0 .\end{cases}
$$




\subsubsection{The source terms}

We refer to [4] for the treatment of the mass exchanges terms $S_{e}\left(X^{n}, \tilde{X}^{n+1}\right)$. For the reaction terms no difficulties occur since the biological variables and what they depend on, light for instance, are also projected on the same Galerkin basis. We write

$$
\int_{z_{\alpha-1 / 2}}^{z_{\alpha+1 / 2}} \int_{x_{i-1 / 2}}^{x_{i+1 / 2}} R\left(x, z, t^{n}\right)=h_{\alpha} \Delta x_{i} R_{i}^{n}
$$

where $R(x, z, t)$ represents one of the three reaction terms we have in the Droop model.

\subsubsection{Properties}

Although we are not going to provide any proof in this section we want to recall some important features of the model used in this paper (see $[4,5,44]$ for the detailed proofs). First of all, under a certain CFL condition which means that the quantity of water leaving the cell during a time step is less than the current water volume of the cell, the water height and the biological concentrations remain non-negative. Second of all, [4] states that a passive tracer satisfies a maximum principle. Finally, we want to specify that a second order scheme in space and time is possible and has been performed in the simulations of Section 4 . The second order in time is achieved through a classical Heun method. We apply a second order in space by a limited reconstruction of the variables [3] based on the prediction of the gradients in each cells, a linear interpolation followed by a limitation procedure.

\section{Simulations}

\subsection{Analytical validation on non trivial steady states}

In this section we show that we can find analytical solutions of the coupled problem, provided that we use Euler equations and a simplified model for biology. We want to emphasize the importance of this part. Validating a numerical code is indeed a complex but highly required task when it comes to non trivial situations. It is clear though that analytical solutions of the whole coupled problem cannot be found. However, we propose here a simplified version of a biological model that would be embedded in free surface Euler equations and for which we can find non trivial steady states.

Let $(\mathrm{u}, \mathrm{w})$ be the following vector field:

$$
\left\{\begin{array}{l}
u(x, z)=\alpha \beta \frac{\cos \left(\beta\left(z-z_{b}\right)\right)}{\sin (\beta H)}, \\
w(x, z)=\frac{\alpha \beta}{\sin (\beta H)^{2}}\left[\sin \left(\beta\left(z-z_{b}\right)\right) \cos (\beta H) \frac{\partial H}{\partial x}+\cos \left(\beta\left(z-z_{b}\right)\right) \sin (\beta H) \frac{\partial z_{b}}{\partial x}\right],
\end{array}\right.
$$

with $\alpha, \beta$ being given constants, $z_{b}(x)$ being known and $H(x)$ being solution of the ordinary differential equation

$$
\frac{\partial}{\partial x}\left(\frac{\alpha^{2} \beta^{2}}{2 \sin ^{2}(\beta H(x))}+g H\right)=-g \frac{\partial z_{b}}{\partial x} .
$$

In [13], the authors show that it is an analytical solution of the Euler system of equations for free surface flows. In order to validate the coupling with biological equations which is performed in this paper, we propose a simplified stationary coupled model.

Let us consider the following scalar field

$$
T(x, z)=\mathrm{e}^{-(H-(z-z b))} .
$$


We can check by simple calculation that $T$ is solution of

$$
\underbrace{\frac{\partial u T}{\partial x}+\frac{\partial w T}{\partial z}}_{\text {advection }}=\underbrace{f(x, z) T(x, z)}_{\text {reaction }}
$$

where

$$
f(x, z)=\alpha \beta \frac{\cos \left(\beta\left(z-z_{b}\right)\right)}{\sin (\beta H)}\left(\frac{\tan \left(\beta\left(z-z_{b}\right)\right)}{\tan (\beta H)}-1\right) \frac{\partial H}{\partial x} .
$$

Clearly, this equation is a simplified and alternate version of a biological model, with advection and reaction terms. Notice that solutions to (4.3) represent non trivial equilibria between advection and reaction terms.

Eventually, we compare analytical and numerical results of the following situation: a given hydrodynamic and biological flow is imposed at the left boundary of a $20 \mathrm{~m}$ long raceway with topography $z_{b}=0.2 \mathrm{e}^{(x-8)^{2}}-$ $0.4 \mathrm{e}^{(x-12)^{2}}$, a given water height is fixed at the right boundary. To solve this analytically we follow [13]: given the topography $z_{b}$, between horizontal coordinates $x=0$ and $x=20$, we recover $H(x)$ and deduce $u(x, z)$ and $w(x, z)$ for $\alpha=0.4$ and $\beta=1.5$ thanks to the above formula $(4.1,4.2)$. Numerically, we impose the analytical flux at the left boundary, the analytical water depth at the right boundary, and run the code for $500 \mathrm{~s}$, with 300 nodes and 20 layers (thus 6000 vertices), while initial conditions were set up to zero for every variable $(u$, $w, T)$. We see in Figure 1 that we recover numerically the hydrological and tracer steady states solutions for this simplified coupled model. Therefore, we consider that our method and our code are likely to produce valid results.

\subsection{Numerical simulations of a raceway}

\subsubsection{Light}

As explained in Section 2, the light intensity at a particular point will depend both on the amount of water above this point (i.e. related to the depth) and on the quantity of encountered chlorophyll (proportional to the concentration in intracellular nitrogen). Additionally, we explain in Section 3 that a Galerkin approximation of the variables is carried out. Hence light is also discretized along the depth by layers. We show in Figure 2 the light profile for different values of $\gamma\left(I^{*}\right)$. Recall that this value is related to the average irradiance perceived by microalgae the day before (photoadaptation phenomenon). The parameters used are exposed in Table 1. We see for any curve the exponential decay. Moreover, this figure shows that the more exposed to light the microalgae were, the more receptive to it they are (no photoinhibition occurs in those ranges of irradiance).

\subsubsection{Paddlewheel}

We aim at representing the kind of raceway shown in Figure 3. In order to model it in 2D, periodic conditions are applied on a rectangular pond containing a paddlewheel in its first half (Fig. 4). The wheel is not modelled physically but is represented by a force that is able to mimic its effect and give the system an equivalent energy. Therefore the following expression is assumed:

$$
\left\{\begin{array}{l}
F_{x}(x, z, t)=F \cos \theta\left(\sqrt{\left(x-x_{\text {wheel }}\right)^{2}+\left(z-z_{\text {wheel }}\right)^{2}} \omega\right)^{2} \\
F_{z}(x, z, t)=F \sin \theta\left(\sqrt{\left(x-x_{\text {wheel }}\right)^{2}+\left(z-z_{\text {wheel }}\right)^{2}} \omega\right)^{2}
\end{array}\right.
$$

where $F$ is a constant, $\theta$ is the angle between the blade and the vertical direction, $\omega=\dot{\theta}$. The force is normal to the blade and depends on the square of the velocity of the point on the blade: the further it is from the center of the wheel, the bigger is the force. Therefore, the energy provided by the wheel is be proportional to the cube of the velocity. To include it in the model, the process is similar to what is explained in Section 3.3: the force is added in the Navier-Stokes equations in order to derive again the multilayer model. This adds a source term in the $\mathrm{x}-$ momentum equation and change the expression of the pressure given by the $\mathrm{z}$-momentum equation. We 


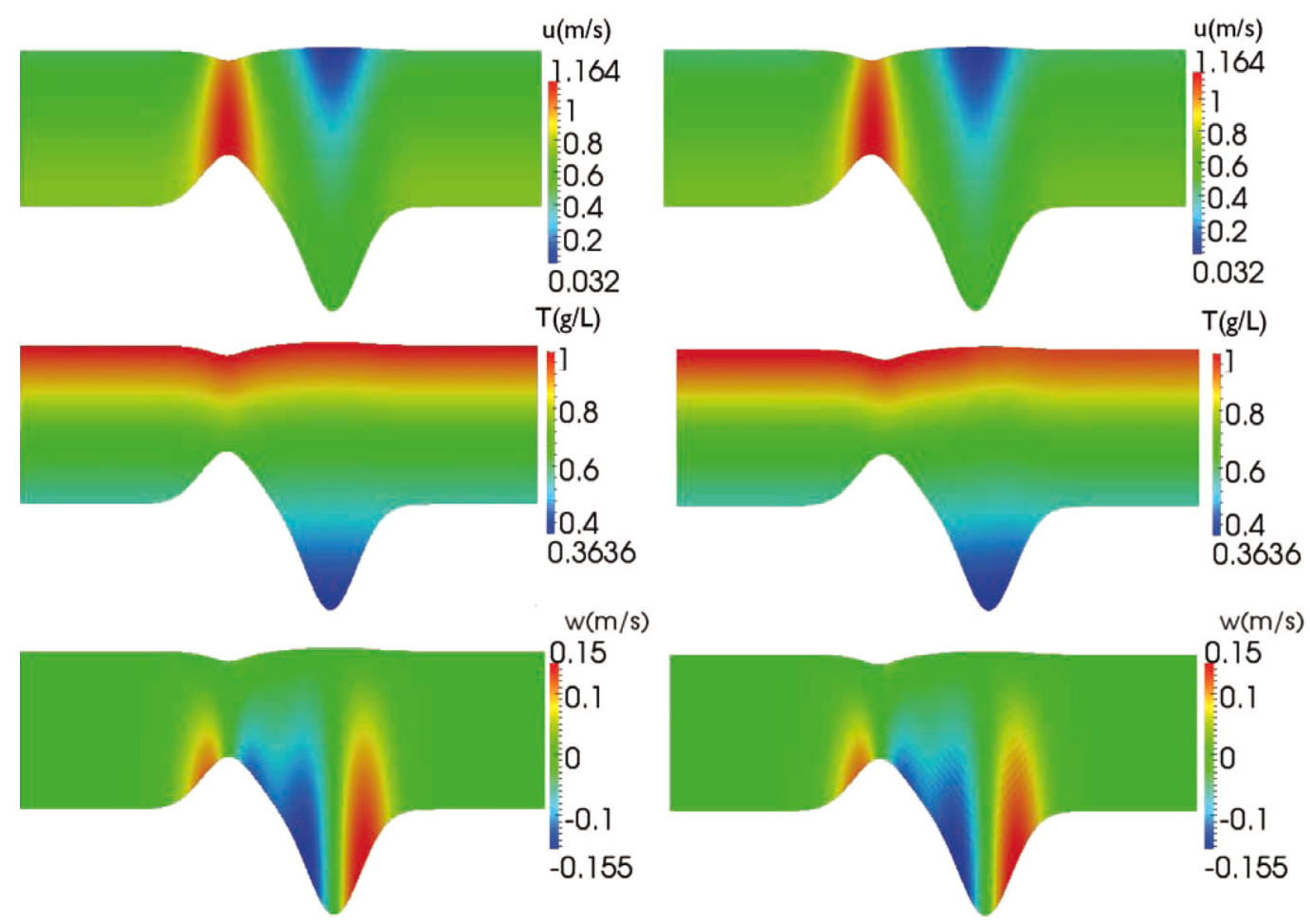

Figure 1. Analytical (left) and numerical (right) steady states solutions for hydrodynamics and tracer in case $\alpha=0.4, \beta=1.5$ and $z_{b}=0.2 \mathrm{e}^{(x-8)^{2}}-0.4 \mathrm{e}^{(x-12)^{2}}$. A given flow is imposed at the left boundary whereas the output boundary condition concerns the water height.

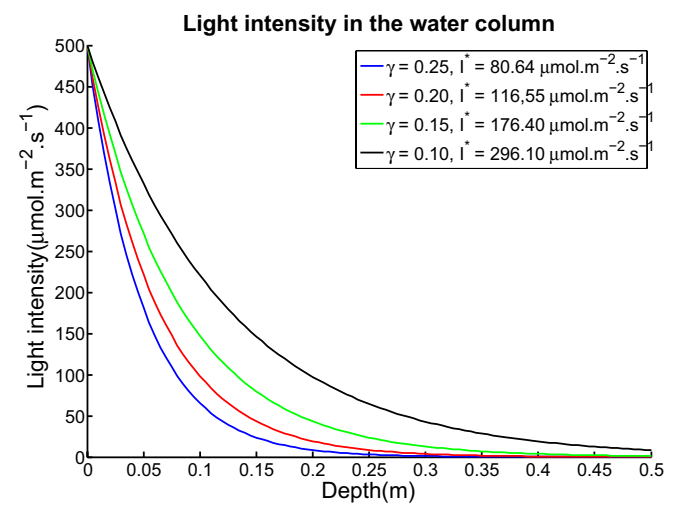

FiguRE 2. Light intensity

TABLE 1. Parameters used for the computation of light. in the water column for a concentration in nitrogen equal to $5.0 \mathrm{gN} \cdot \mathrm{m}^{-3}$. The figure shows the exponential decay of light with depth for different values of $\gamma\left(I^{*}\right)$.

\begin{tabular}{|c|c|c|}
\hline Parameter & Value & Unit \\
\hline$I_{0, \max }$ & 500 & $\mu \mathrm{mol} \cdot \mathrm{m}^{-2} \cdot \mathrm{s}^{-1}$ \\
$\mathrm{a}$ & 16.2 & $\mathrm{~m}^{2} \cdot \mathrm{gChl}^{-1}$ \\
$\mathrm{~b}$ & 0.087 & $\mathrm{~m}^{-1}$ \\
$C^{2}$ & 5.0 & $\mathrm{gN} \cdot \mathrm{m}^{-3}$ \\
\hline
\end{tabular}




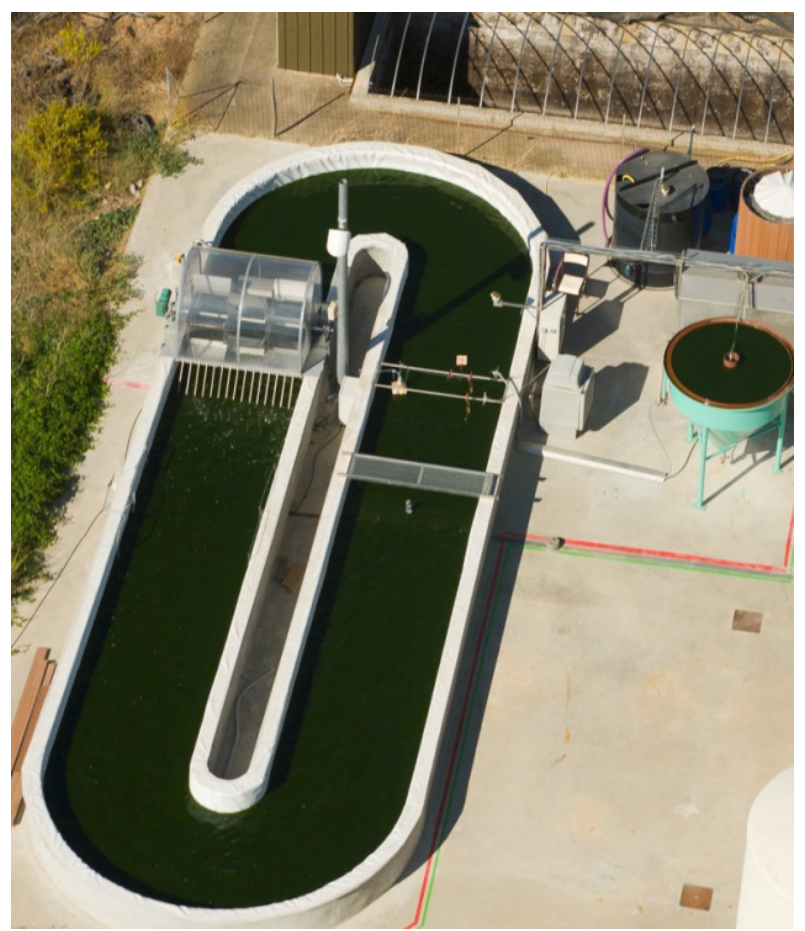

Figure 3. A typical raceway for cultivating microalgae. Notice the paddlewheel which mixes the culture suspension. Picture from INRA (ANR Symbiose project).

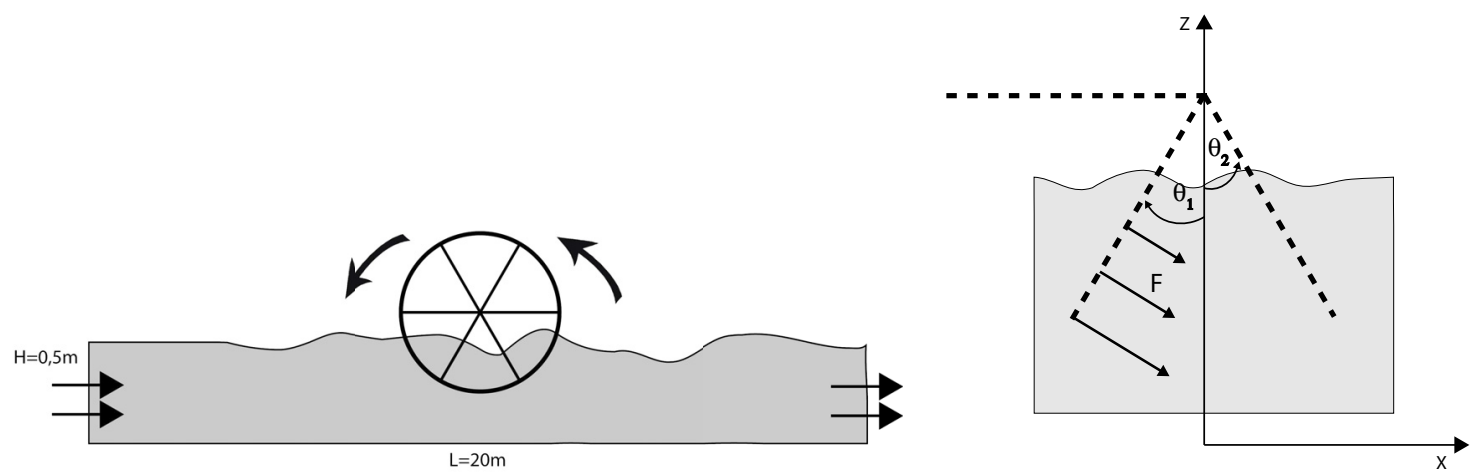

FiguRE 4. Left: Raceway and wheel dimensions and positions. Right: Outlook of the force applied to model the effect of the paddlewheel, supposed to be located in the first half of the raceway, with maximum efficiency at the end of the blade.

remind the reader that non-hydrostatic terms have not been taken into account in the derivation of the model (though it is described in [44]). The obtained results in Figure 5 let us think that this may be appropriate to model the paddlewheel effect.

We performed several simulations for different paddlewheel angular velocities. We use a raceway with length $20 \mathrm{~m}$ and height $0,5 \mathrm{~m}$. We show in Figure 5 that we are able to capture realistic hydrodynamics: a laminar flow of reasonable horizontal speed far from the wheel and a turbulent flow close to it. Concerning the hydrodynamics 

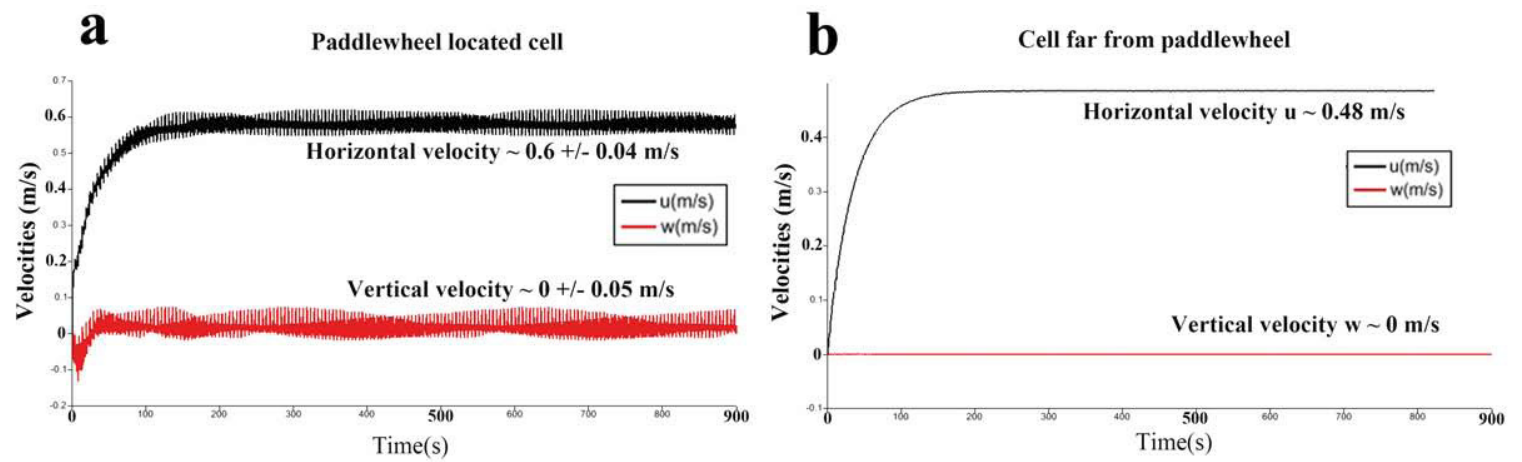

Figure 5. (a): Velocities along vertical and horizontal axis in a cell located near from the wheel rotating at angular speed $\omega=0.8 \mathrm{rad} / \mathrm{s}$, with a force magnitude of $F=10 \mathrm{~N} . \mathrm{m}^{-3}$. The flow is very turbulent. (b): Velocities along vertical and horizontal axis in a cell located far from the wheel. An asymptotic value of $0.48 \mathrm{~m} \cdot \mathrm{s}^{-1}$ is reached.

parameters, we take into account horizontal and vertical viscosity $\left(\mu=0.001 \mathrm{~m}^{2} . s^{-1}\right)$ and a Navier-type bottom friction $\left(\kappa=0.01 \mathrm{~m} \cdot \mathrm{s}^{-1}\right)$. Besides, $\omega=0.85 \mathrm{rad} / \mathrm{s}$.

In order to have a first idea of what is happening in the fluid, we add a tracer which is advected and slightly diffused. Figure 6 illustrates the effect of the wheel on the mixing. In particular, after several minutes, the pond seems well homogenized (here again $\omega=0.85 \mathrm{rad} / \mathrm{s}$ ).

\subsection{Results}

Eventually, we performed numerical simulations of the whole coupled model explained in Section 2 and discretized in Section 3. We use the same raceway with length $20 \mathrm{~m}$ and height $0,5 \mathrm{~m}$. The height is chosen such that at the bottom of the pond, the respiration rate is close to the growth rate. We perform several 20 days simulations for different agitations, different initial conditions and we compare them to reference simulations i.e. without paddlewheel. The parameters used for the simulation (concerning the biological system) are exposed in Table 2. The relevant details of every simulation are in Table 3. The results are depicted in Figure 7.

The initial concentrations of particulate and dissolved nitrogen are the same for the six simulations. The initial microalgal carbon concentration varies (the initial internal nitrogen quota is therefore changing as well), and different agitation velocities are tested. The plots represent the average concentrations in the raceway (see local concentration comparison between upper and bottom layer in Fig. 8). The carbon curves (Fig. 7a) show in every situation that agitation leads to better productivity. This is explained by the lack of nutrients in the upper layers at some point. However, regarding the initial internal quota (Fig. 7b), the time when agitation actually enhances productivity varies. This phenomena is due to the fact that the internal nutrient pool, if not filled enough(low quota $q$ ), will tend to increase further by absorbing more external nitrogen. Thus leading two main consequences: the extracellular nutrient concentration diminishes and the intracellular nitrogen increases. Since chlorophyll is positively correlated with the latter biological variable, the light can not penetrate so deep anymore. We point out that the model is not able to differentiate between several agitation velocities. Ideas to explain and overcome this limitation are suggested in Section 5.

From these series of simulations we can conclude that our model is capable of reproducing coherent results, in the hydrodynamical part (adequate asymptotic velocity, turbulences near the wheel and laminar flow far from it) as well as in the biological concentrations. Nevertheless, we can only consider those results as preliminary, since no quantitative comparison with any data has been provided. Future work would include adaptation of the biological model given the hydrodynamical results obtained in this study. Moreover, other variables should be added to the system. For instance temperature, which could have a non negligible effect, sedimentation etc. 


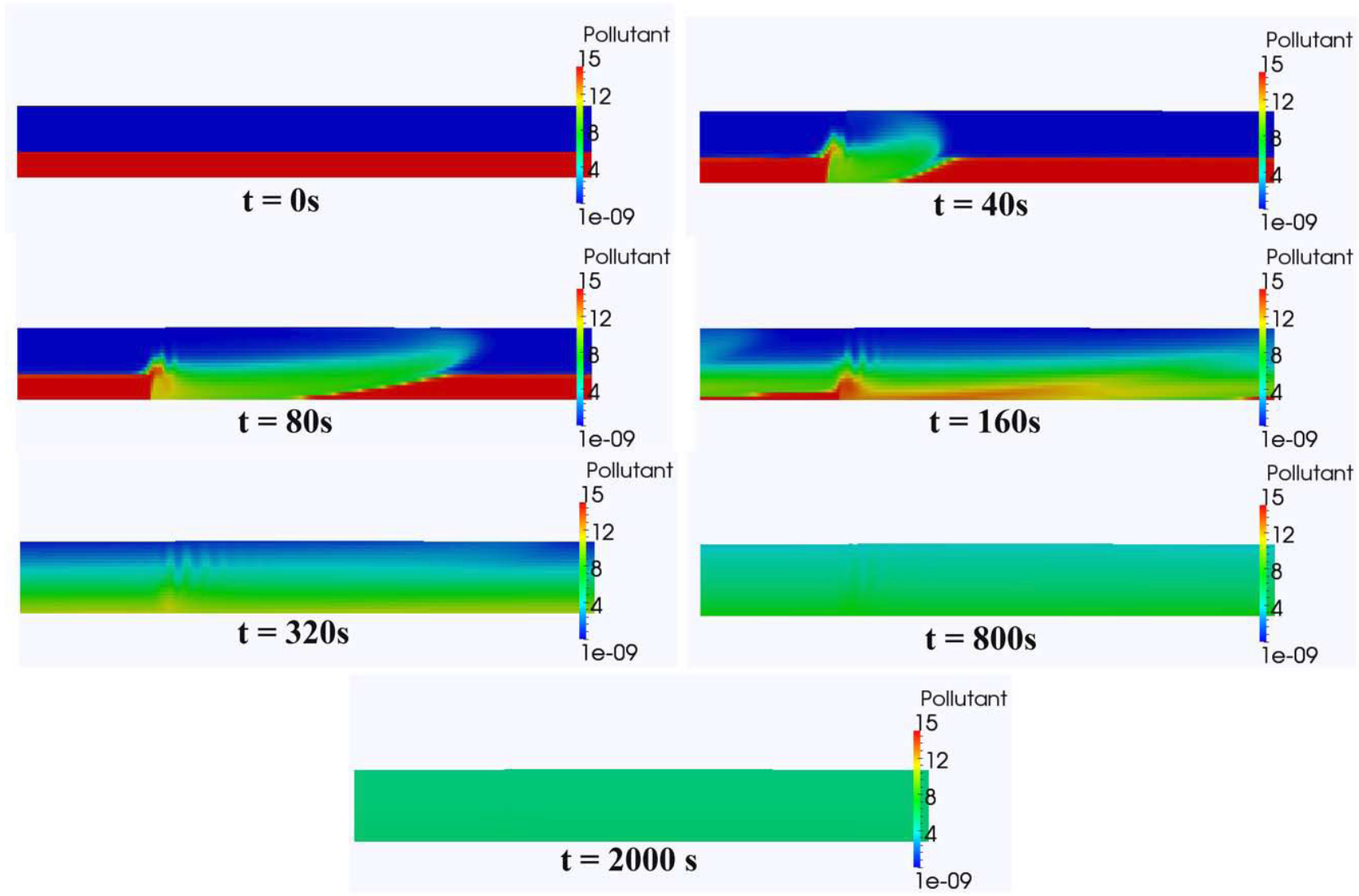

FiguRE 6. Snapshots of the tracer concentration in a raceway set into motion by a paddlwheel of angular velocity $\omega=0.85 \mathrm{rad} / \mathrm{s}$. It is clear that after several minutes, the raceway is totally homogeneous. Therefore, the paddlewheel has indeed the required effect on the mixing.

Finally, experimental data should help us calibrate more accurately the raceway parameters and extend it to three dimensions.

\section{LAGRANGIAN APPROACH}

So far, we have based our model on biological kinetics accounting for the photosynthesis process in conditions of light and nutrient limitations. We have used an extended version of the Droop model $[10,19,20]$, but other models could have been used, differing by their level of details $[7,8,23]$. These models have been developped and experimentally validated for static conditions, i.e. for conditions where light was constant or slowly varying. However, the present study illustrates the fact that, due to hydrodynamics, each cell experiments a succession of light and dark phases, depending if it is close to surface or to the bottom. Such flashing light phenomenon has already been highlighted with photobioreactors [39]. Now the key question is to determine if the chosen model, with time scales in the range of hours, efficicently reproduces the photosynthesis dynamics at the cell scale, or if a more sophisticated model, accounting for the fast time scales of photosynthesis is required. Indeed, some models represent faster biological phenomena. For example, the Han model [28] represents the dynamics of the photosystems with time scales ranging from milliseconds to minutes. Such model would, of course, be more appropriate to account for photosynthesis in the context of rapid light fluctuations, with strong potential 
TABle 2. Parameters for the simulations.

\begin{tabular}{|l|c|c|}
\hline Parameter & Value & Unit \\
\hline$\tilde{\mu}$ & 1.7 & day $^{-1}$ \\
$Q_{0}$ & 0.050 & ${\mathrm{gN} . \mathrm{gC}^{-1}}^{-1}$ \\
$Q_{l}$ & 0.25 & $\mathrm{gN} \cdot \mathrm{gC}^{-1}$ \\
$K_{i I}$ & 295 & $\mu \mathrm{mol} \cdot \mathrm{m}^{-2} \cdot \mathrm{s}^{-1}$ \\
$K_{s I}$ & 70 & $\mu \mathrm{mol} \cdot \mathrm{m}^{-2} \cdot \mathrm{s}^{-1}$ \\
$\bar{\lambda}$ & 0.073 & $\mathrm{gN} \cdot \mathrm{gC}^{-1} \cdot \mathrm{day}^{-1}$ \\
$K_{s}$ & 0.0012 & $\mathrm{gN} \cdot \mathrm{m}^{-3}$ \\
$\mathrm{R}$ & 0.0081 & day \\
$I_{0, \max }$ & 500 & $\mu \mathrm{mol} \cdot \mathrm{m}^{-2} \cdot \mathrm{s}^{-1}$ \\
$\gamma\left(I^{*}\right)$ & 0.25 & $\mathrm{gChl} \mathrm{gN}^{-1}$ \\
$\mathrm{a}$ & 16.2 & $\mathrm{~m}^{2} \cdot \mathrm{gChl}^{-1}$ \\
$\mathrm{~b}$ & 0.087 & $\mathrm{~m}^{-1}$ \\
\hline
\end{tabular}

TABLE 3. Initial conditions and final concentrations for the set of 6 simulations we performed. The differences lie on the one hand on the fact that the water is agitated or not and on the other hand on the initial carbon concentration, which changes the internal quota (nitrogen concentration is always equal to $5\left(\mathrm{~g} \cdot \mathrm{m}^{-3}\right)$.

\begin{tabular}{|l|cccccc|}
\cline { 2 - 7 } \multicolumn{1}{c|}{} & Simu 1 & Simu 2 & Simu 3 & Simu 4 & Simu 5 & Simu 6 \\
\hline$C_{0}^{1}\left(\mathrm{~g} \cdot \mathrm{m}^{-3}\right)$ & 25 & 25 & 50 & 50 & 83 & 83 \\
$\left(\frac{C^{2}}{C^{1}}\right)_{0}\left(\mathrm{gN} \cdot \mathrm{gC}^{-1}\right)$ & 0.2 & 0.2 & 0.1 & 0.1 & 0.06 & 0.06 \\
$C_{0}^{3}\left(\mathrm{~g} \cdot \mathrm{m}^{-3}\right)$ & 5 & 5 & 5 & 5 & 5 & 5 \\
Agitation & no & yes & no & yes & no & yes \\
$C_{f}^{1}\left(\mathrm{~g} \cdot \mathrm{m}^{-3}\right)$ & 60 & 74 & 79 & 103 & 100 & 129 \\
$\left(\frac{C^{2}}{C^{\mathrm{I}}}\right)_{f}\left(\mathrm{gN} \cdot \mathrm{gC}^{-1}\right)$ & 0.115 & 0.120 & 0.110 & 0.085 & 0.087 & 0.065 \\
$C_{f}^{3}\left(\mathrm{~g} \cdot \mathrm{m}^{-3}\right)$ & 3.77 & 0.0 & 0.0 & 0.0 & 0.0 & 0.0 \\
\hline
\end{tabular}

impact on the physiology [48]. As a consequence, it is now crucial to determine the typical light pattern received by a single cell. It is worth noting that such an information can only be obtained by simulation, since it would be very complex to measure experimentally the light along the trajectory of a microalgae. A Lagrangian approach derived from the previous model can lead to the reconstruction of cell trajectories. From this information, it is then possible to derive the light signals that microalgal cells undergo. We show hereafter that the velocity of the wheel does not influence much the light quantity perceived by an algae in a certain period of time, but it changes the way it receives it.

In order to follow the position of a particle, we simply need to integrate the following equation. If $M(t)$ is the position of particle $M$ at time t, then we have:

$$
\frac{\mathrm{d} M(t)}{\mathrm{d} t}=v(M(t), t)
$$

where $v(x, t)$ is the eulerian velocity field at position $x$, time $t$. We do not add to those particles any Brownian motion that would refer to the diffusion of biological concentrations at the macroscopic scale. Therefore, more realistic Lagrangian trajectories should look more noisy than what we get but the general behaviour is well represented. 

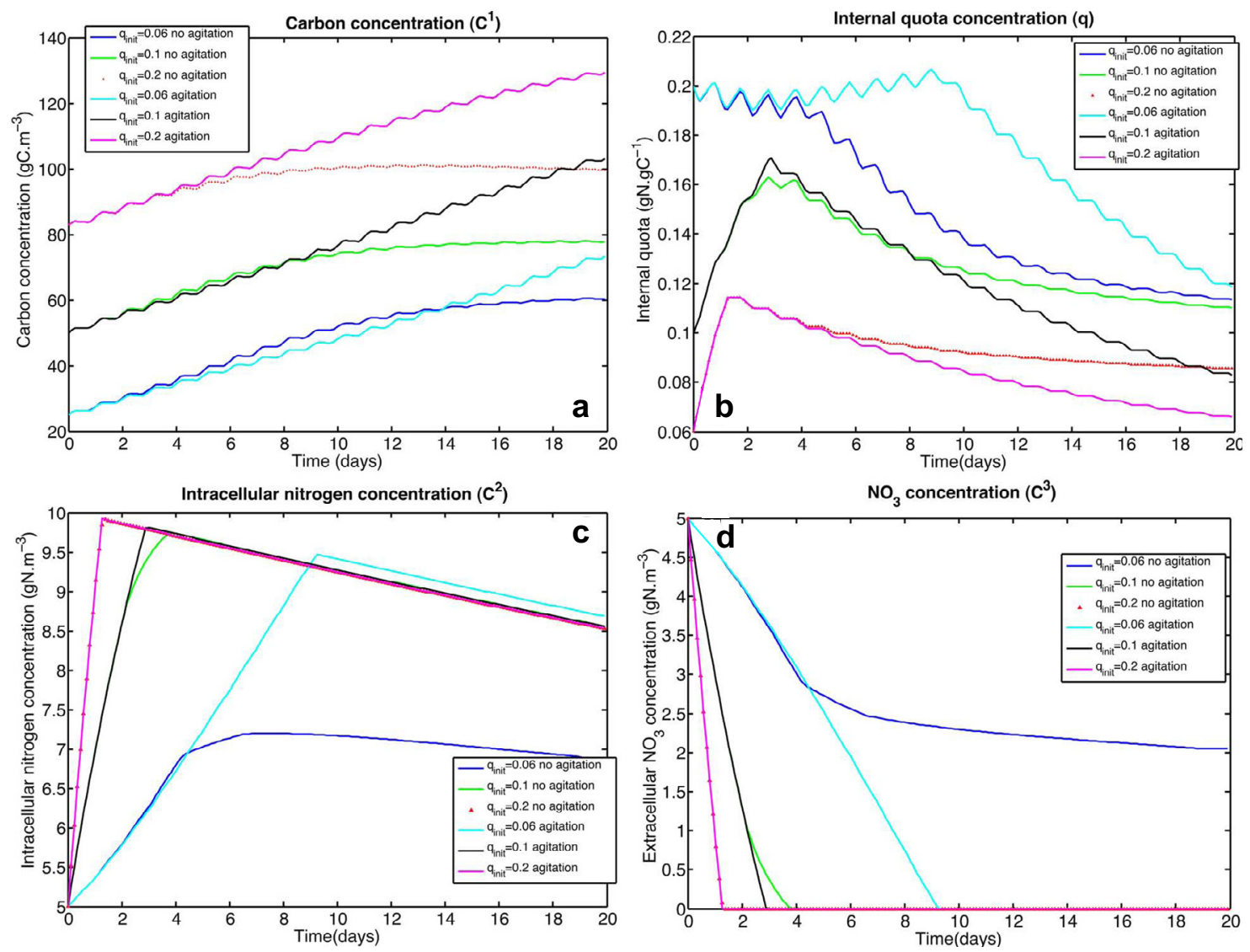

FIgURE 7. (a): Carbon concentration; (b): Internal quota $q$; (c): nitrogen concentration; (d): substrate concentration $\left(\mathrm{NO}_{3}\right)$. Those plots illustrate the average concentrations in the raceway for 6 simulations. Three were carried out without agitation, and the other three had the agitation term. In each situation, agitation, leading to homogenization leads to a better productivity. However, for certain initial conditions, the improvement is quite slow (after several days), since the biological variables do not evolve as quickly as hydrodynamics does.

For several angular velocities, we perform a one hour simulation and build the trajectories afterwards. One hundred particles are equally distributed in the raceway along the depth dimension. Figure 9 shows the distribution of particles against the percentage of time spent under more than $50 \%$ of the incident light (we will call it high enlightenment). We notice that the wheel velocity has an influence on the number of particles which never undergo high enlightenment (38 particles over 100 for $\omega=0.5$ and only 13 particles over 100 for $\omega=1.0$ ). But globally, the particles are distributed around $20 \%$ of their time under high enlightenment. This is what we can expect from a good mixing since the light is exponentially decreasing and getting $50 \%$ of the incident light means being in the first $10 \mathrm{~cm}$ from the surface of the raceway (over $50 \mathrm{~cm}$ ).

In Figure 10 we plot different indicators for eight velocities, established during a one hour simulation. First of all, Figure 10a illustrates the average velocity at the end of the simulation. It turns out to lie in realistic ranges $\left(0.2 \mathrm{~m} . \mathrm{s}^{-1}\right.$ to $\left.0.7 \mathrm{~m} . \mathrm{s}^{-1}\right)$. Second of all Figure 10b represents the average proportion of time spent for any particle under high enlightenment. From this curve we deduce that the global percentage of high enlightenment may not vary much between $\omega=0.5$ and $\omega=1$. The mixing is indeed well carried on (as shown in previous section) and in average, the particles have the same history. Finally, Figure 10c depicts the number of times 

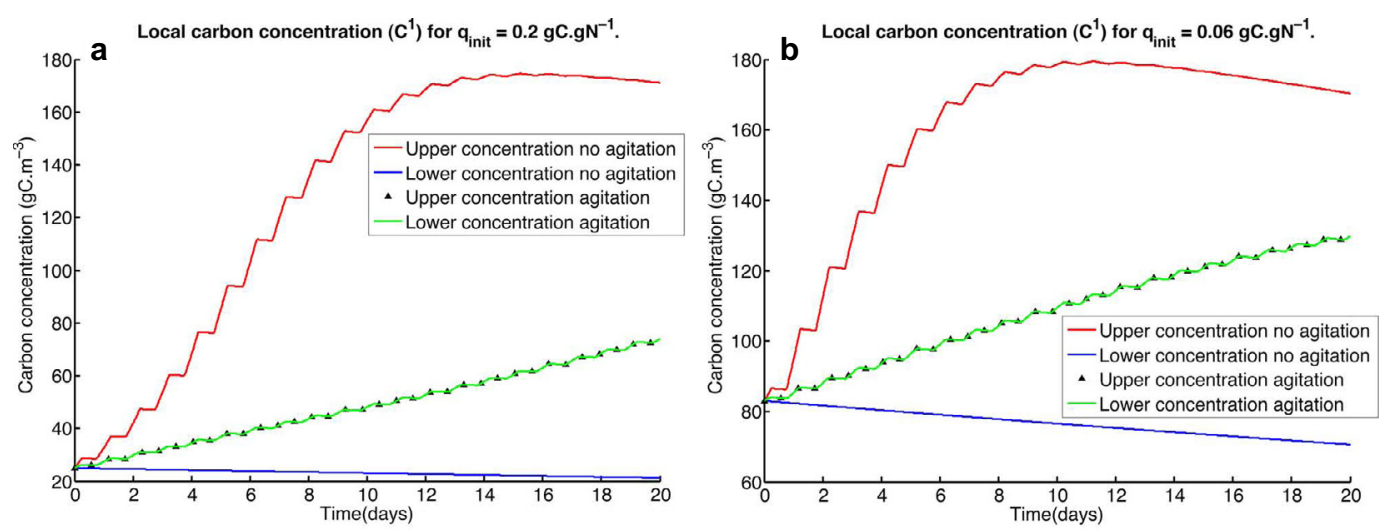

FiguRE 8. (a): Local carbon concentration when $q_{\text {init }}=0.2 \mathrm{gN} \cdot \mathrm{gC}^{-1}$, in the bottom and upper layer, with and without agitation. (b): Local carbon concentration when $q_{\text {init }}=0.06 \mathrm{gN} \cdot \mathrm{gC}^{-1}$, in the bottom and upper layer, with and without agitation. We see clearly the homogenization due to agitation. In both cases, we also see that the bottom layer when not agitated does not vary so much, which means that we are close to the point when respiration compensates growth.

$\omega=0.5$

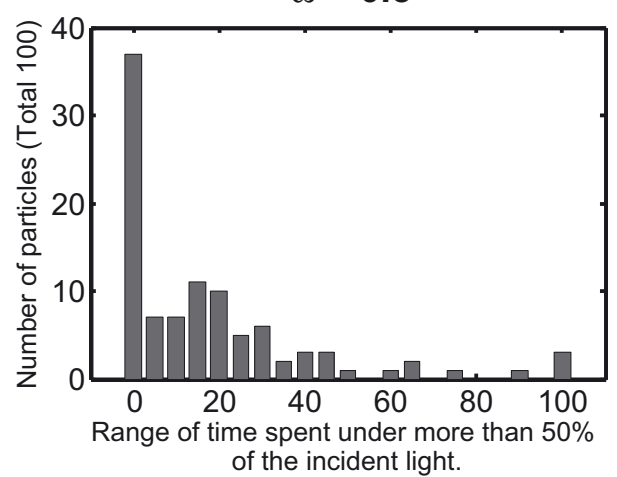

$\omega=0.85$

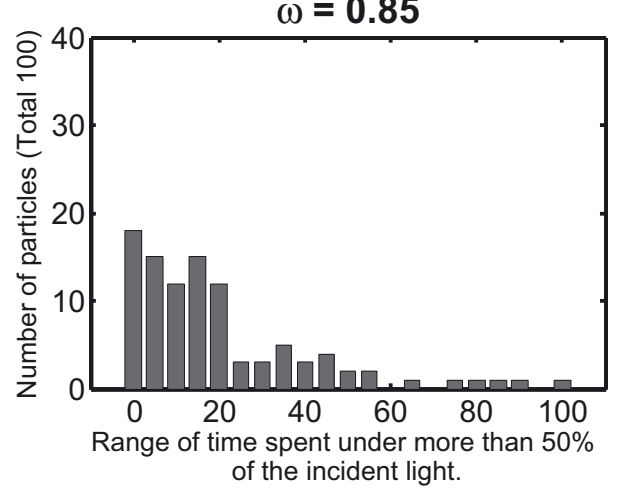

$\omega=0.75$

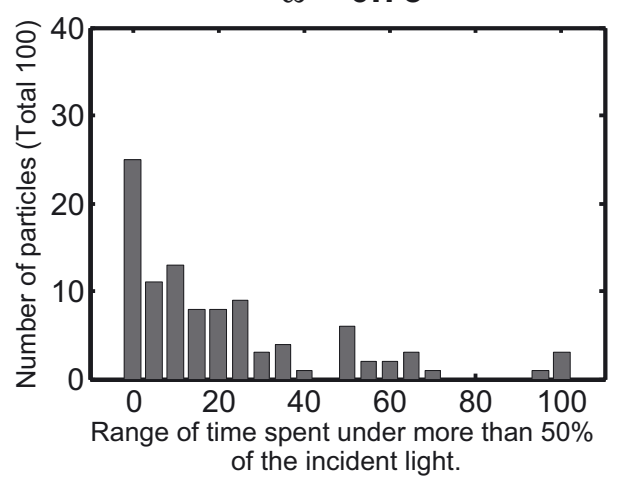

$\omega=1.0$

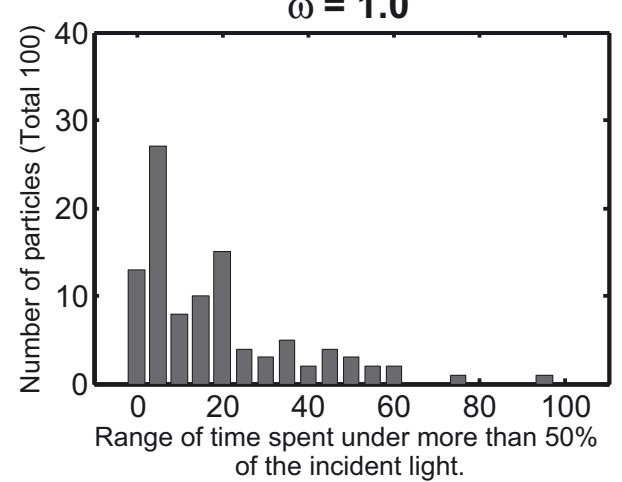

Figure 9. Number of particles for each class of enlightenment for four wheel angular veloci-

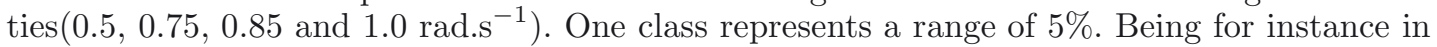
the class 20\%-25\% means that the particle spent between 20 and $25 \%$ of her time under hight enlightenment (more than $50 \%$ of the incident light). 

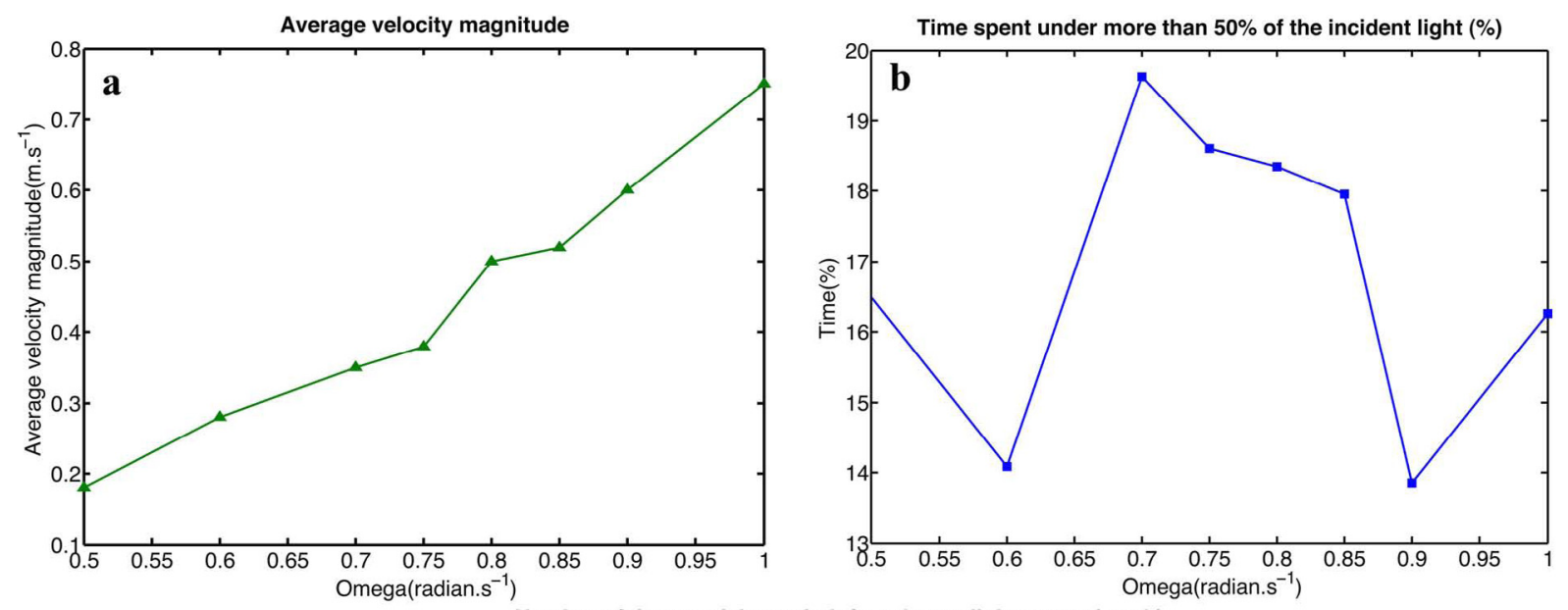

Number of time particles switch from low enlightenment $\left(<0.5 \mathrm{I}_{0}\right)$

to high enlightenment $\left(>0.5 \mathrm{I}_{0}\right)$

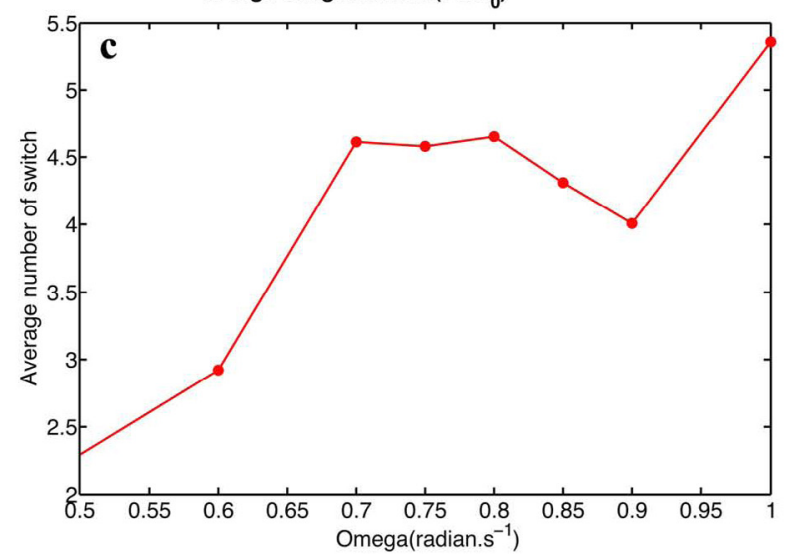

FiguRE 10. (a): Average velocity after one hour for 8 velocities. (b): Percentage of time spent under more than $50 \%$ of $I_{0}$. (c): Number of times a particles switches from a situation where it perceived less than $50 \%$ of light intensity to a situation where it perceives more during one hour.

the particles switch from low enlightenment to high enlightenment during one hour. This last indicator gives insights about the duration of the high enlightenment periods. The greater it is, the shorter but more numerous were the high light instants (the particles switches more often).

Finally, the trajectories of three particles are depicted in Figure 11a. From those trajectories we can extract two important informations. First of all, between two passages around the paddlewheel, the particle seems to stay at constant depth, thus enforcing the fact that the flow is laminar apart from the wheel. Second of all, we clearly see that the depth of the particle is suddenly modified by the wheel, giving rise to abrupt changes in the enlightenment. Figure 11b shows the light received by those three particles in the case where the average intracellular nitrogen concentration is $5 \mathrm{gN} . \mathrm{m}^{-3}$ and $\gamma\left(I^{*}\right)=0.1 \mathrm{gChl}_{\mathrm{gN}}{ }^{-1}$ (high irradiance the day before the simulation).

Figure 2 shows that light intensity is very low in the 20 deepest centimeters. Regarding our results on Figure 11, we assume that microalgae should spend one lap at high light and then several laps at low light. Since the asymptotic velocity is in the range $0.3 \mathrm{~m} \cdot \mathrm{s}^{-1}-0.8 \mathrm{~m} \cdot \mathrm{s}^{-1}$, a whole lap is done in the order of a minute. Therefore, algae are faced to light changes with a time scale in the range of ten minutes. As regards our modeling 

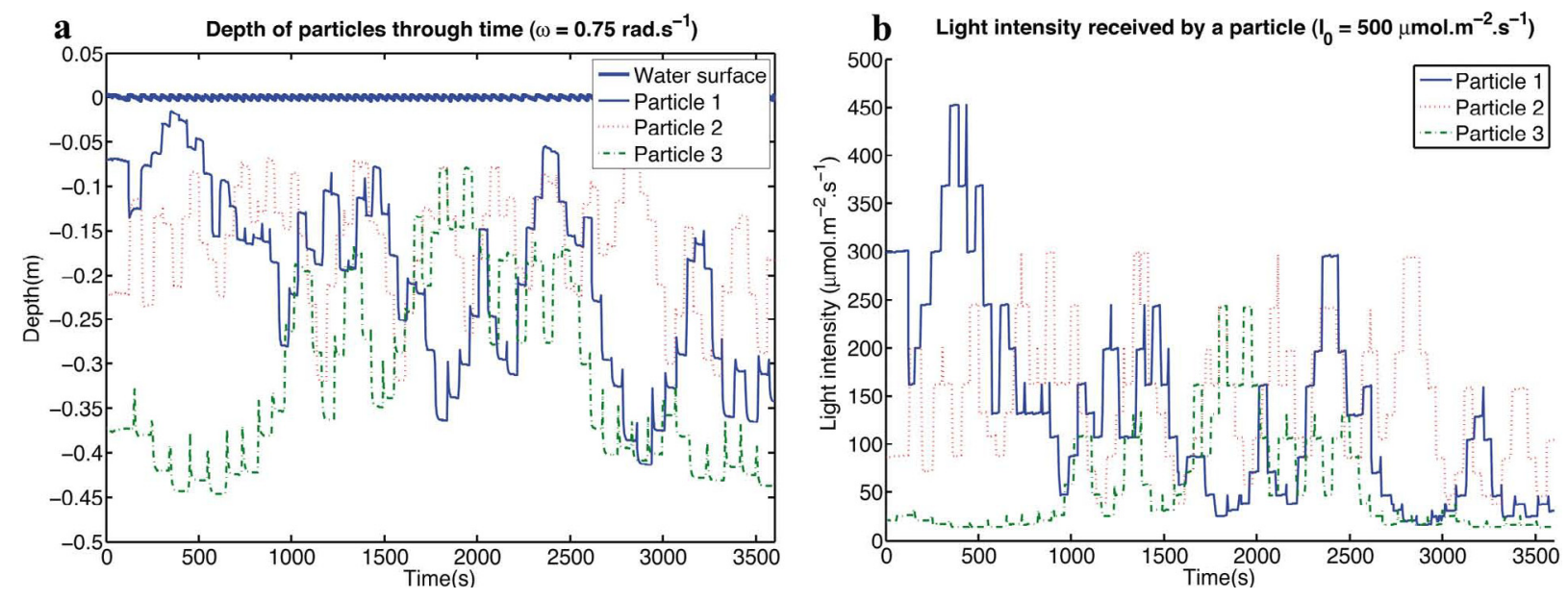

FIGURE 11. (a):Trajectories of three particles during the simulations. The large curve represents the water surface at the middle of the raceway. The other plot is the height of a given particle through time. The algae undergo sudden changes of depth every time it meets the wheel.(b): Perceived light from the microalgae. Particles are subject to even greater irradiance changes since the light is exponentially decaying.

problem, this tells us that a biological model valid for these fast time scale could lead to more precise results. Additional experiments forcing microalgae with typical light signal deduced from Figure 2 must therefore been carried out and support a microalgae modelling at fast time scale [22].

\section{Conclusion}

In this paper we derive a new model coupling hydrodynamics to biology in two dimensions. It provides new insights to better understand and represent this nonlinear and non stationary complex process. Except closed to the paddlewheel, the multilayer model seems to adequately represent the vertical heterogeneity characterizing the agitated raceway. Notice that with our approach, we are able to provide analytical solutions which validated its numerical integration. The results show that water agitating through the paddlewheel has an effect on the growth of algae, particularly because of the lack of nutrients at the surface versus the lack of light around the raceway's floor. One of the outcome of this work is the identification of realistic light signals to which microalgae are faced. Lab scale experiments will be performed to assess the impact of such high frequency light signals on microalgae. It is worth remarking that similar works have been carried out for photobioreactor $[38,39]$, leading to the identification of much faster time scales in the range of the second. Of course, it is possible to improve the model around the wheel by relaxing the hydrostatic approximation. A robust an efficient scheme for the discretization of the non-hydrostatic terms is under development. Though theoretical results and first experiments have already been carried out $[15,16]$, this model has not yet been widely validated and requires the coding of much complex numerical schemes. Likewise a 3D model is necessary to take into account the effects of the bend of the hydroynamics. The comparison with experimental data would be of great relevance to better calibrate the hydrodynamics, and later on improve the biological predictions of the model. It is clear however that many parameters need to be taken into account in order to increase the model prediction capacity, for instance temperature. It was here considered as a passive tracer, only advected and diffused, with no particular effect on the biology (temperature does not appear in the growth or respiration rate). The sunlight effect on water temperature will be taken into account in a next stage since it deeply affects microalgae growth. Moreover, some microalgae species do not swim in the water and tend to sediment. This property could increase the beneficial effect of the wheel compared to a situation where the wheel is very slow or absent. Computational 
time is another issue which has to be improved in order to use the model e.g. for process optimal design. Up to now, schemes we are developing are only explicit. We are therefore constrained with a restrictive CFL condition. Improvement towards implicit schemes is also a future concern.

Acknowledgements. The authors would like to thank the support of INRIA ARC Nautilus (http://www-roc.inria.fr/ bang/Nautilus/?page=accueil) together with the ANR Symbiose project (http://www.anr-symbiose.org).

\section{REFERENCES}

[1] E Audusse, Modelisation hyperbolique et analyse numerique pour les ecoulements en eaux peu profondes. Ph.D. thesis. Université Pierre et Marie Curie - Paris VI (2004).

[2] E. Audusse and M.-O. Bristeau, Transport of pollutant in shallow water flows: A two time steps kinetic method. ESAIM: M2AN 37 (2003) 389-416.

[3] E. Audusse and M.-O. Bristeau, A well-balanced positivity preserving second-order scheme for shallow water flows on unstructured meshes. J. Comput. Phys. 206 (2005) 311-333.

[4] E. Audusse, M.-O. Bristeau, M. Pelanti and J. Sainte-Marie, Approximation of the hydrostatic Navier-Stokes system for density stratified flows by a multilayer model. Kinetic interpretation and numerical validation. J. Comput. Phys. 230 (2011) $3453-3478$.

[5] E. Audusse, M.-O. Bristeau, B. Perthame and J. Sainte-Marie, A multilayer saint-venant system with mass exchanges for shallow water flows. Derivation and numerical validation. ESAIM: M2AN 45 (2011) 169-200.

[6] E. Audusse, F. Bouchut, M.-O. Bristeau, R. Klein and Be. Perthame, A fast and stable well-balanced scheme with hydrostatic reconstruction for shallow water flows. SIAM J. Sci. Comput. 25 (2004) 2050-2065.

[7] S.-D. Ayata, M. Lévy, O. Aumont, A. Sciandra, J. Sainte-Marie, A. Tagliabue and O. Bernard, Phytoplankton growth formulation in marine ecosystem models: should we take into account photo-acclimation and variable stochiometry in oligotrophic areas? To appear in J. Marine Syst.

[8] M. Baklouti, F. Diaz, C. Pinazo, V. Faure and B. Queguiner, Investigation of mechanistic formulations depicting phytoplankton dynamics for models of marine pelagic ecosystems and description of a new model. Progr. Oceanogr. 71 (2006) 1-33.

[9] A.-J.-C. Barré de Saint-Venant, Théorie du mouvement non permanent des eaux, avec application aux crues des rivières et à l'introduction des marées dans leur lit. Comptes Rendus des Séances de l'Académie des Sciences, Paris 73 (1871) 147-154.

[10] O. Bernard, Hurdles and challenges for modelling and control of microalgae for co2 mitigation and biofuel production. $J$. Process Control 21 (2011) 1378-1389.

[11] O. Bernard and J.-L. Gouzé, Transient behavior of biological loop models, with application to the Droop model. Math. Biosci. 127 (1995) 19-43.

[12] O. Bernard and J.-L. Gouzé, Global qualitative behavior of a class of nonlinear biological systems: application to the qualitative validation of phytoplankton growth models. Artif. Intel. 136 (2002) 29-59.

[13] A.-C. Boulanger and J. Sainte-Marie, Analytical solutions for the free surface hydrostatic euler equations. Submitted to Nonlinearity (2011).

[14] J.-F. Bourgat, P. Le Tallec, F. Mallinger, B. Perthame, Y. Qiu, C. boltzmann and navier-stokes, Research Report RR-2281, Projet MENUSIN. INRIA (1994).

[15] M.-O. Bristeau and J. Sainte-Marie, Derivation of a non-hydrostatic shallow water model; Comparison with Saint-Venant and Boussinesq systems. DCDS(B) 10 (2008) 733-759.

[16] M.-O. Bristeau, N. Goutal and J. Sainte-Marie, Numerical simulations of a non-hydrostatic shallow water model. Comput. Fluids 47 (2011) 51-64.

[17] V. Casulli, A semi-implicit finite difference method for non-hydrostatic, free-surface flows. Int. J. Numer. Methods Fluids 30 (1999) 425-440.

[18] Y. Chisti, Biodiesel from microalgae. Biotech. Adv. 25 (2007) 294-306.

[19] M.R. Droop, Vitamin $\mathrm{B}_{12}$ and marine ecology. IV. the kinetics of uptake growth and inhibition in Monochrysis lutheri. J. Mar. Biol. Assoc. 48 (1968) 689-733.

[20] M.R. Droop, 25 years of algal growth kinetics, a personal view. Botanica Marina 16 (1983) 99-112.

[21] R.C. Dugdale, Nutrient limitation in the sea: dynamics, identification and significance. Limnol. Oceanogr. 12 (1967) 685-695.

[22] S. Esposito, V. Botte, D. Iudicone and M. Ribera d'Alcala, Numerical analysis of cumulative impact of phytoplankton photoresponses to light variation on carbon assimilation. J. Theor. Biol 261 (2009) 361-371.

[23] R.J. Geider, H.L. MacIntyre and T.M. Kana, A dynamic regulatory model of phytoplanktonic acclimation to light, nutrients, and temperature. Limnol Oceanogr 43 (1998) 679-694.

[24] J.-F. Gerbeau and B. Perthame, Derivation of viscous saint-venant system for laminar shallow water; numerical validation. Discrete Contin. Dyn. Syst. Ser. B 1 (2001) 89-102.

[25] J.U. Grobbelaar, C.J. Soeder and E. Stengel, Modeling algal productivity in large outdoor cultures and waste treatment systems. Biomass 21 (1990) 297-314.

[26] H. Guterman, A. Vonshak and S. Ben-Yaakov, A macromodel for outdoor algal mass production. Biotechnol. Bioengineer. 35 (1990) 809-819. 
[27] B.P. Han, Photosynthesis-irradiance response at physiological level: a mechanistic model. J. Theoret. Biol. 213 (2001) $121-127$.

[28] B.P. Han, A mechanistic model of algal photoinhibition induced by photodamage to photosystem-ii. J. Theoret. Biology 214 (2002) 519-527.

[29] J.-M. Hervouet, Hydrodynamics of Free Surface Flows: Modelling With the Finite Element Method. John Wiley and Sons (2007).

[30] D.L. Huggins, R.H. Piedrahita and T. Rumsey, Analysis of sediment transport modeling using computational fluid dynamics (cfd) for aquaculture raceways. Aquacult. Engrg. 31 (2004) 277-293.

[31] D.L. Huggins, R.H. Piedrahita and T. Rumsey, Use of computational fluid dynamics (cfd) for aquaculture raceway design to increase settling effectiveness. Aquacult. Engrg. 33 (2005) 167-180.

[32] S.C. James and V. Boriah, Modeling algae growth in an open-channel raceway. J Comput. Biol. 17 (2010) 895-906.

[33] B. Khobalatte and B. Perthame, Maximum principle on the entropy and minimal limitations for kinetic schemes. Research Report RR-1628, Projet MENUSIN. INRIA (1992).

[34] K. Lange and F.J. Oyarzun, The attractiveness of the Droop equations. Math. Biosci. 111 (1992) 261-278.

[35] H.-P. Luo and M.H. Al-Dahhan, Analyzing and modeling of photobioreactors by combining first principles of physiology and hydrodynamics. Biotechnol. Bioengineer. 85 (2004) 382-393.

[36] F.B. Metting, Biodiversity and application of microalgae. J. Indust. Microbiol. Biotechnol. 17 (1996) $477-489$.

[37] J. C. H. Peeters and P. Eilers, The relationship between light intensity and photosynthesis: a simple mathematical model. Hydrobiol. Bull. 12 (1978) 134-136.

[38] I. Perner, C. Posten and J. Broneske, Cfd-aided optimization of a plate photobioreactor for cultivation of microalgae. Chemie Ingenieur Technik 74 (2002) 865-869.

[39] I. Perner-Nochta and C. Posten, Simulations of light intensity variation in photobioreactors. J. Biotechnol. 131 (2007) $276-285$.

[40] B. Perthame, Kinetic formulation of conservation laws. Oxford lecture series in mathematics and its applications. Oxford University Press (2002).

[41] J. Pruvost, L. Pottier and J. Legrand, Numerical investigation of hydrodynamic and mixing conditions in a torus photobioreactor. Chemical Engineer. Sci. 61 (2006) 4476-4489.

[42] L. Rodolfi, G.C. Zittelli, N. Bassi, G. Padovani, N. Biondi, G. Bonini and M.R. Tredici, Microalgae for Oil: Strain Selection, Induction of Lipid Synthesis and Outdoor Mass Cultivation in a Low-Cost Photobioreactor. Biotechnol. Bioeng. 102 (2009) $100-112$

[43] R. Rosello Sastre, Z. Coesgoer, I. Perner-Nochta, P. Fleck-Schneider and C. Posten, Scale-down of microalgae cultivations in tubular photo-bioreactors - a conceptual approach. J. Biotechnol. 132 (2007) 127-133.

[44] J. Sainte-Marie, Vertically averaged models for the free surface euler system. derivation and kinetic interpretation. Math. Models Methods Appl. Sci. 21 (2011) 459-490.

[45] A. Sciandra and P. Ramani, The limitations of continuous cultures with low rates of medium renewal per cell. J. Exp. Mar. Biol. Ecol. 178 (1994) 1-15.

[46] A. Sukenik, P.G. Falkowski and J. Bennett. Potential enhancement of photosynthetic energy conversion in algal mass culture. Biotechnol. Bioengineer. 30 (1987) 970-977.

[47] A. Sukenik, R.S. Levy, Y. Levy, P.G. Falkowski and Z. Dubinsky, Optimizing algal biomass production in an outdoor pond: a simulation model. J. Appl. Phycol. 3 (1991) 191-201.

[48] C. Vejrazka, M. Janssen, M. Streefland and R.H. Wijffels, Photosynthetic efficiency of chlamydomonas reinhardtii in flashing light. Biotechnol. Bioengineer. 108 (2011) 2905-2913.

[49] R.H. Wijffels and M.J. Barbosa, An outlook on microalgal biofuels. Science 329 (2010) 796-799.

[50] P.J.B. Williams and L.M.L. Laurens, Microalgae as biodiesel and biomass feedstocks: Review and analysis of the biochemistry, energetics and economics. Energy Environ. Sci. 3 (2010) 554-590. 